\title{
The IDA cell separation pathway connects developmental and defense responses
}

\author{
Vilde Olsson', Elwira Smakowska-Luzan ${ }^{2 \S}$, Maike Breiden ${ }^{3 \S}$, Peter Marhavy ${ }^{4}$, Rebecca \\ Schneeweiss $^{2}$, Youssef Belkhadir ${ }^{2}$, Rüdiger Simon ${ }^{3}$ and Melinka A. Butenko ${ }^{1^{*}}$
}

${ }^{1}$ Section for Genetics and Evolutionary Biology, Department of Biosciences, University of Oslo, 0316

Oslo, Norway

${ }^{2}$ Gregor Mendel Institute (GMI), Austrian Academy of Sciences, Vienna Biocenter (VBC), Dr Bohr-

Gasse 3, 1030 Vienna, Austria

${ }^{3}$ Institute for Developmental Genetics and Cluster of Excellence on Plant Sciences, Heinrich Heine University, Universitätsstraße 1, 40225 Düsseldorf, Germany

${ }^{4}$ Department of Plant Molecular Biology, University of Lausanne, 1015 Lausanne, Switzerland

${ }^{\S}$ Equal contribution

*Corresponding author; email: m.a.butenko@ibv.uio.no 


\begin{abstract}
The abscission of floral organs and emergence of lateral roots in Arabidopsis is regulated by the peptide ligand INFLORESCENCE DEFICIENT IN ABSCISSION (IDA) and the receptor protein kinases HAESA (HAE), HAESA-LIKE 2 (HSL2) and members of the SOMATIC EMBRYOGENESIS RECEPTOR KINASE (SERK) family. These cell separation processes lead to induction of defense-associated genes to protect against pathogen invasion. However, the molecular coordination between abscission and immunity has not been thoroughly explored. Here we show that IDA induces a receptor-dependent release of cytosolic calcium and an extracellular release of reactive oxygen species which are signatures of defense responses. IDA promotes heteromerization between HSL2 and RECEPTOR LIKE KINASE 7 (RLK7), a receptor that enhances immunity and pathogen responses, and utilizes this novel signaling module to regulate the expression of defense-associated genes. We propose a molecular mechanism by which IDA drives specific immune response in cells destined for separation to guard them from pathogen attack.
\end{abstract}

\title{
Introduction
}

In plants, organ separation or abscission, involves cell separation between specialized abscission zone (AZ) cells and enables the removal of unwanted or diseased organs in response to endogenous developmental cues or environmental stimuli. Precise cell separation is also essential during plant development and growth, including emergence of new lateral root (LR) primordia, root cap sloughing, formation of stomata and radicle emergence during germination ${ }^{1}$. In Arabidopsis thaliana (Arabidopsis), a peptide mediated signaling system ensures the correct spatial and temporal abscission of sepals, petals and stamens. Floral organ abscission is regulated by the genetically redundant receptor protein kinases HAESA (HAE) and HAESA-LIKE 2 (HSL2) that bind the secreted INFLORESCENCE DEFICIENT in ABSCISSION (IDA) peptide promoting receptor association with members of the co-receptor SOMATIC EMBRYOGENESIS RECEPTOR KINASE (SERK) family ${ }^{2-5}$. Plants carrying mutations in both the HAE and HSL2 genes, the IDA gene or higher order combinations of the $S E R K$ genes fail to activate an intracellular signaling pathway including a mitogen-activated protein kinase (MAPK) cascade and members of the transcription factor family KNOTTED-LIKE FROM ARABIDOPSIS THALIANA (KNAT) ${ }^{2,3,6}$. A deficiency in the IDA signaling pathway impedes the expression of genes encoding secreted cell wall remodeling and hydrolase enzymes thus hindering floral organs to abscise ${ }^{2,3,5,7-10}$. Interestingly, like for other signal transduction pathways where phylogenetically related families of peptides and receptors regulate similar developmental processes, components of the IDA signaling system control different cell separation events during Arabidopsis development. IDA signaling through HAE and HSL2 regulates 
cell wall remodeling genes in the endodermal, cortical and epidermal tissues overlaying the LR primordia during LR emergence ${ }^{8}$. In addition, IDA-LIKE 1 (IDL1), one of the eight members in the IDL peptide family ${ }^{11}$ that shares a conserved C-terminal domain which in IDA is processed to a functional 14 amino acid hydroxylated peptide ${ }^{12,13}$, signals through HSL2 to regulate cell separation during root cap sloughing ${ }^{14}$.

Plant cell walls act as physical barriers against pathogenic invaders and as thus the cell-wall processing and remodeling that occurs during abscission and other cell separation events provides an entry point for phytopathogens ${ }^{15}$. Indeed it has been shown that AZ cells undergoing cell separation express defense genes that have altered expression in the hae hsl2 mutant and it has been proposed that they function in protecting the AZ from infection after abscission has occurred ${ }^{9,16}$. As such, mediators of cell-wall alteration during plant development could have an effect on plant pathogenicity and substantiating this, it has been shown that pathogenic bacteria utilize components of the abscission signaling pathway to induce pectin degrading enzymes and ease Arabidopsis rosette leaf colonization ${ }^{17}$. Furthermore, plants can contain bacterial infection by shedding diseased organs ${ }^{18,19}$. and in Arabidopsis, cauline leaves infected with Pseudomonas syringae are abscised by utilizing the same signaling pathway that regulates floral organ abscission ${ }^{19}$.

From the induction of defense related genes during abscission to the use of abscission regulating signals to inhibit bacterial infections, it is apparent that there is a tight interplay between plant defense and abscission. We sought to understand how plant defense programs are unfolded against the backdrop of coordinated cell separation events and found that IDA modulates plant immune signaling. Here, we report that a range of biotic and abiotic signals can induce IDA expression. We demonstrate that IDA is able to activate an arm of the plant immune system that involves the detection of microbe-/danger-associated molecular patterns (MAMPs/DAMPs). This effect is modulated by HSL2 and RECEPTOR-LIKE KINASE 7 (RLK7). Finally, we establish that IDA promotes a direct physical interaction between RLK7 and HSL2. Thus, plants utilize IDA-mediated signaling to coordinate distinct, yet intertwined, cellular functions. We propose that the IDA peptide originally played a more general function in plant innate immunity and later developed a specified role in regulating cell separation and in protection of exposed cells during the abscission process.

\section{Results}

\section{Expression of $I D A$ in response to abiotic and biotic stresses}

Transgenic plants containing the $\beta$-glucuronidase $(G U S)$ gene under the control of the IDA promoter ( $p I D A: G U S$ ) have previously been reported to exhibit GUS expression in AZ tissue at stages of flower development where there is weakening of the cell walls of AZ cells and in cells overlaying newly 
formed LR primordia ${ }^{7,8}$. To investigate the expression of the IDA gene in response to MAMPs pIDA:GUS seedlings were exposed to the bacterial derived epitope flg22 and fungal chitin. Compared to the control seedlings, the seedlings exposed to MAMPs showed a marked increase in GUS expression (Fig. 1a and Supplementary Fig. 1a). As described earlier ${ }^{8}$, the GUS signal in seedlings grown on untreated media was observed in epidermal and cortical cells covering the LR primordia during the emergence of a new LR (Fig. 1a and Supplementary Fig. 1a); while in flg22 and chitin treated seedlings the intensity of the GUS signal was enhanced (Fig. 1a and Supplementary Fig. 1a). To determine the activity of GUS, 4-methylumbelliferyl $\beta$-D-glucuronide (4-MUG) was used as a substrate. Upon 4-MUG hydrolysis by GUS the fluorochrome 4-methyl umbelliferone (4-MU) is produced and can be quantified by the emitted fluorescence ${ }^{20}$. Untreated, flg22 treated and chitin treated pIDA:GUS seedlings were incubated with 4-MUG and the fluorescence emitted by 4-MU was measured and normalised per sample. A significant increase in fluorescence was observed for flg22 and chitin treated samples compared to the untreated control, verifying the observed enhancement of GUS signal in the micrographs (Fig. 1b and Supplementary Fig. 1b).

Immune signaling in plants in response to flg22 is initiated by the activation of the FLAGELLINSENSITIVE 2 (FLS2) receptor upon peptide perception ${ }^{21,22}$. flg22 treatment of pIDA:GUS seedlings in the $f l s 2$ mutant background compared to untreated $p I D A: G U S$ fls 2 seedlings showed no significant difference in GUS activity (Fig. 1a,b) indicating that the flg22 induced IDA expression is FLS2 dependent. Interestingly, PIP1, an endogenous DAMP peptide known to amplify the immune response triggered by flg 22 and pathogen resistance through RLK ${ }^{23}$, could also significantly induce pIDA:GUS expression; while the inactive IDA peptide, IDA $^{\Delta \mathrm{N} 69} 4,12, \operatorname{did} \operatorname{not}($ Fig. 1a,b). As for flg22, the PIP1 induced IDA expression was dependent on the peptide being perceived by its cognate receptor as no enhancement of pIDA:GUS expression was detected in PIP1 treated pIDA:GUS rlk7 seedlings (Fig. 1a,b).

Spatial IDA expression was also monitored upon induced abiotic stress. When pIDA:GUS seedling were treated with the osmotic agent mannitol or exposed to salt stress by $\mathrm{NaCl}$, a similar significant increment in GUS signal was observed as for seedlings subjected to biotic stimuli (Supplementary Fig. 1a,b). As both mannitol and $\mathrm{NaCl}$ lower the water potential of the medium they simulate what happens in drying soil. Under normal growing conditions Arabidopsis does not display cauline leaf abscission, however, when experiencing drought followed by re-watering, cauline leaf abscission is triggered concurrent with an increase in IDA transcript levels in the leaf $\mathrm{AZs}^{24}$. Given that abiotic stress induces abscission in Arabidopsis it is conceivable that biotic effectors like flg22 induce IDA expression as a requisite for pathogen triggered cell separation; alternatively increased IDA levels 
could trigger a defense response. To explore this avenue further the role of the IDA peptide in plant immunity was investigated.

\section{mIDA triggers expression of defense-associated marker genes associated with innate immunity}

Transcriptional reprogramming mostly mediated by WRKY transcription factors takes place during MAMP-triggered immunity (MTI) and is essential to mount an appropriate host defense response ${ }^{25}$. The expression of MYB51, a WRKY target gene, has previously been shown to increase in response to flg22 ${ }^{26}$ resulting in biosynthesis of the secondary metabolite indolic glucosinolates which plays a role in defense. To investigate whether IDA could regulate a similar set of genes induced by flg22 we looked at roots expressing the promoter-driven nuclear localized YFP lines of MYB51 $\left(\text { pMYB51:YFP }{ }_{N}\right)^{27}$ treated with a highly active synthetic IDA peptide ${ }^{12}$ (from here on referred to as mIDA). The fluorescent signal of the reporter was analyzed in the root tip/meristematic zone and an enhanced expression of MYB51 was predominantly detected in the meristematic zone after 7 hours (h) of mIDA treatment compared to a non-treated control (Fig. 1c). Fluorescent nuclei were also detected in the root tip and LR cap in response to mIDA (Fig. 1c). RT-qPCR experiments from 7 days-old seedlings treated with mIDA confirmed the mIDA induced expression of $p M Y B 51: Y F P_{N}$ with significantly higher levels of expression after 1 and $12 \mathrm{~h}$ when compared to untreated tissue (Fig. 1d). Moreover, comparison of mIDA induction with the elicitor-triggered response of $p M Y B 51: Y F P_{N}$ to flg22 showed similar temporal expression in the root (Supplementary Fig. 2). To further explore the role of mIDA as an inducer of immunity we selected well-established defense-associated marker genes and analyzed the ability of mIDA to induce the expression of FLG22-INDUCED RECEPTORLIKE KINASE1 (FRK1), a specific and early immune-responsive gene activated by multiple MAMPs 28,29, the endogenous danger peptide ELICITOR PEPTIDE 3 (PEP3) ${ }^{30}$ and WRKY33, a key transcriptional regulator of defense responses ${ }^{25,31}$ by RT-qPCR. Seven days-old Col-0 wild-type (WT) seedlings treated with mIDA had elevated expression levels of FRK1 and PEP3 compared to untreated control samples (Fig. 1d). In accordance with previous reports, where the expression of FRK1 and PEP3 was monitored in response to bacterial elicitors ${ }^{29,30}$, the significant increase of expression was rapid and observed already after $1 \mathrm{~h}$ of mIDA treatment (Fig. 1d). After $12 \mathrm{~h}$, FRKI expression in mIDA treated tissue was not significantly different from expression in untreated tissue; while PEP3 expression in mIDA treated tissue remained elevated after $12 \mathrm{~h}$ but was significantly lower than the expression detected after $1 \mathrm{~h}$ of mIDA treatment (Fig. $1 \mathrm{~d}$ ). The expression of WRKY33, a defense gene known to be required for resistance towards necrotrophs, was not increased in mIDA treated seedlings after $1 \mathrm{~h}$ but showed a 2 -fold increase when compared to untreated tissue after $12 \mathrm{~h}$ (Fig. $1 \mathrm{~d}$ ). The temporal delay in induction of WRKY33 compared to FRK1 and PEP3 is comparable 
to previous reports for leaf samples inoculated with Botrytis cinerea ${ }^{31}$. Together, these data show that IDA can trigger a rapid, albeit transient increase in the expression of key defense genes.

\section{mIDA triggers defense-associated cellular responses}

Next we sought to address whether IDA could induce cellular responses associated with early MTI events. In addition to the transcriptional reprogramming associated with MTI, the rapid production of reactive oxygen species (ROS) burst and the elevation of cytosolic calcium-ion concentrations $\left(\left[\mathrm{Ca}^{2+}\right]_{\mathrm{cyt}}\right)$ is a conserved signaling output in immunity across kingdoms ${ }^{32,33}$. To test the ability of mIDA to trigger an increase in $\left[\mathrm{Ca}^{2+}\right]_{\text {cyt }}$ and to study mIDA-induced $\mathrm{Ca}^{2+}$ dynamics we performed $\mathrm{Ca}^{2+}$ imaging of 10 days-old roots expressing the cytosolic localized fluorescent $\mathrm{Ca}^{2+}$ sensor, RGECO1. We detected a clear increase in $\left[\mathrm{Ca}^{2+}\right]_{\text {cyt }}$ after application of $1 \mu \mathrm{M}$ mIDA (Fig. 2a,b and Movie 1). Regions of interest (ROIs) along the whole root were analyzed for signal intensity values and revealed that the $\mathrm{Ca}^{2+}$ signal was composed of two main waves. The signal initiated in the root meristematic zone from where it spread toward the elongation zone and root tip, a second wave was observed in the meristematic zone continuing with $\mathrm{Ca}^{2+}$ spikes (Fig. $2 \mathrm{~b}$ and Movie 1). The signal amplitude was at maximum within the elongation zone and decreased as the signal spread (Fig. 2a,b and Movie 1). Interestingly, when comparing the $\mathrm{Ca}^{2+}$ dynamics in mIDA treated roots to those treated with flg22 we observed striking differences in the onset and distribution of the $\mathrm{Ca}^{2+}$ signal. Analysis of roots treated with $1 \mu \mathrm{M}$ flg22 showed that the $\mathrm{Ca}^{2+}$ signal initiated in the root elongation zone from where it spread toward the meristematic zone as a single wave and that the signal amplitude was at maximum within the elongation zone and decreased as the signal spread (Supplementary Fig. $3 \mathrm{a}, \mathrm{b}$ and Movie 2). The spatial onset of the $\mathrm{Ca}^{2+}$ response in mIDA treated roots correlated with the spatial expression pattern of $p M Y B 51: Y F P_{N}$ in mIDA treated roots (Fig. 1c) which differed from that previously reported for the flg22 elicited response ${ }^{27}$. These observations indicate differences in tissue specificity of immune responses between mIDA and flg22 which are likely to depend on the cellular distribution of their cognate receptors. Indeed, when investigating the promoter activity of the $H A E$ and $H S L 2$ receptors by cloning the promoters fused to the nuclear localized YFP-derived fluorophore Venus protein (pHAE:Venus-H2B and pHSL2:Venus-H2B) we observed fluorescent nuclei in the lateral root cap, root tip and root meristem in pHSL2:Venus-H2B lines and in the epidermis and stele of the elongation zone in $p H A E$ :Venus-H2B lines (Fig. 2c). This Indicated that the mIDA induced $\left[\mathrm{Ca}^{2+}\right]_{\text {cyt }}$ was dependent on receptors known to bind IDA ${ }^{3,4,12,}$.

To investigate the mIDA dependency of HAE and HSL2 for $\left[\mathrm{Ca}^{2+}\right]_{c y t}$ increase a cytosolic localized Aequorin-based luminescence $\mathrm{Ca}^{2+}$ sensor (Aeq) was introduced into the hae hsl2 mutant background. The Aeq sensor was chosen due to transgene silencing of the R-GECO1 reporter in hae hsl2. The 
mIDA triggered increase in $\left[\mathrm{Ca}^{2+}\right]_{\text {cyt }}$ was completely abolished in hae hsl2 Aeq seedlings compared to Aeq expressing seedlings (Fig. 2d), indicating that the response observed is receptor dependent. The mIDA dependent increase in $\left[\mathrm{Ca}^{2+}\right]_{\text {cyt }}$ was also peptide dose-dependent with a half-maximal efficient concentration ( $\left.\mathrm{EC}_{50}\right)$ of $500 \mathrm{nM}$ (Supplementary Fig. 4). To explore whether an increase in $\left[\mathrm{Ca}^{2+}\right]_{\text {cyt }}$ could also be triggered in response to mIDA in flowers, we monitored $\left[\mathrm{Ca}^{2+}\right]_{\text {cyt }}$ changes in flowers expressing the Aeq sensor at different developmental stages (Fig. 2e). Interestingly, only flowers at a the stage where there is an initial weakening of the cell walls of AZ cells showed an increase in $\left[\mathrm{Ca}^{2+}\right]_{\text {cyt. }}$. Flowers treated with mIDA prior to cell wall loosening showed no increase in luminescence (Supplementary Fig. 5a,b), indicating that the mIDA triggered $\mathrm{Ca}^{2+}$ release in flowers correlates with the onset of the abscission process and the increase in HAE and HSL2 expression at the $\mathrm{AZ}^{34}$.

ROS production is tightly connected to increased $\left[\mathrm{Ca}^{2+}\right]_{\text {cyt }}$ as $\mathrm{Ca}^{2+}$ partially activates members of the NOX family of NADPH oxidases (RBOHs) which are key producers of ROS ${ }^{32}$. We have previously shown that mIDA elicits the production of a ROS burst in Nicotiana benthamiana (N.benthamiana) leaves transiently expressing $H A E$ or $H S L 2{ }^{12}$. To investigate whether mIDA could also elicit a ROS burst in Arabidopsis, a luminol-dependent ROS assay on hae hsl2 mutant rosette leaves expressing a functional full-length HAE receptor with a YFP fluorescent tag from a constitutive cauliflower mosaic virus (CaMV) 35S promoter and a YFP fluorescent tag (35S:HAE-YFP) (Supplementary Fig. 6a,b) was used. hae hsl2 35S:HAE-YFP leaf discs treated with mIDA emitted extracellular ROS whereas the response was not observed in WT leaves (Fig. 2f). Furthermore, by staining WT flowers undergoing cell separation (position 6, see Supplementary Fig. 5a) with $\mathrm{H}_{2}$ DCF-DA, a highly fluorescent indicator for reactive oxygen intermediates in cells, we could detect a strong fluorescent signal in AZ cells. The fluorescent signal was absent from AZ cells in hae hsl2 mutant flowers (Fig. $3 a)$, indicating that the ROS production, like the $\left[\mathrm{Ca}^{2+}\right]_{c y t}$ increase, was receptor dependent. Transcriptional data revealed that $R B O H D$ and $R B O H F$ are highly expressed in the floral $\mathrm{AZs}{ }^{16}$ and we therefore set out to investigate if ROS production in AZ cells was dependent on RBOHD and RBOHF and whether these NADPH oxidases were necessary for organ separation. The presence of $\mathrm{H}_{2}$ DCF-DA staining in the AZs and the normal floral abscission of rbohd rbohf mutant flowers indicated that neither ROS production nor cell separation was dependent on $R B O H D$ and $R B O H F$ (Fig. 3a,b). These results are in stark contrast to what has previously been reported, where cell separation was shown to be dependent on $R B O H D$ and $R B O H F{ }^{35}$. Subtle differences in floral abscission are difficult to detect and to unequivocally determine a difference in floral abscission between a mutant and WT it is necessary to quantify the force needed to remove petals from the receptacle of gradually older flowers along the inflorescence. This is performed by a stress transducer 
that measures the petal breakstrength (pBS) ${ }^{5}$. To conclude whether $R B O H D$ and $R B O H F$ were necessary for the abscission process we monitored floral organ abscission in rbohd rbohf mutants by pBS. Measurements showed that at the stage where initial cell wall remodeling and a marked reduction in pBS is seen in WT plants (floral position 5, see Supplementary Fig. 5a) ${ }^{7}$, there was a significant lower value for the rbohd rbohf mutant than WT, indicating premature cell wall loosening (Fig. 3c). Furthermore, rbohd rbohf mutant petals abscised one position earlier than WT as it was not possible to measure the pBS of rbohd rbohf mutant petals at position 6 (Fig. 3c). Different T-DNA alleles of $R B O H D$ and $R B O H F$ were crossed and phenotypical investigation of double mutants did not show any plants with a delay in abscission (Supplementary Fig. 7). In conclusion, we found no RBOHD and RBOHF dependency for cell separation during floral abscission or for ROS production, indicating that other RBOHs or peroxidases are responsible for the HAE HSL2 dependent ROS release in AZs. The ROS production detected by $\mathrm{H}_{2}$ DCF-DA staining in the rbohd rbohf mutant flowers (Fig. 3a) is independent from the RBOHD and F-dependent lignification in AZs ${ }^{35}$ and we suggest that this ROS burst may play a role in defense.

\section{Receptors associated with innate immunity are expressed in cells undergoing cell separation} Given that PIP1 and flg22 induce IDA expression by signaling through their cognate receptors RLK7 and FLS2, respectively (Fig. 1a,b) we investigated the promoter activity of these receptors in tissues where IDA and IDL peptides regulate cell separation and compared it to that of HAE and HSL2. The promoters were cloned and fused to the nuclear localized YFP-derived fluorophore Venus protein (pRECEPTOR:Venus-H2B). The nuclear localization facilitates the cell-specific detection of the fluorescent signal and to analyse the expression pattern of pRLK7:Venus-H2B, pHSL2:Venus-H2B, pHAE:Venus-H2B and pFLS2:Venus-H2B during LR emergence, roots from 7 days-old seedlings were stained with propidium iodide and investigated using a confocal laser scanning microscope. As reported previously, promoter activity was detected for $H S L 2$ and $H A E$ in cells at the base of the LR during LR emergence and the promoter of HSL2 had prominent activity in the LRP (Fig. 4a). Both the promoters of $R L K 7$ and FLS2 were active in cells surrounding the LRP (Fig. 4a), where also IDA is expressed (Fig. 1a). In the root tip, fluorescent nuclei could be observed in the LR cap for pRLK7:Venus-H2B and pHSL2:Venus-H2B lines, while no fluorescent nuclei were observed for pHAE:Venus-H2B or pFLS2:Venus-H2B lines (Fig. 2c and supplementary Fig. 8).

In Arabidopsis, floral organ abscission occurs shortly after anthesis and in WT plants the organs abscise at position 7 (counted from the first flower with visible white petals at the top of the inflorescence, see Supplementary Fig. 5a) ${ }^{5}$. The promoter activity of the receptors in AZ cells undergoing cell separation was investigated in flowers at position 6 by removing the floral organs. 
Fluorescent nuclei could be observed in the petal, sepal and anther AZs for all pRECEPTOR:Venus$H 2 B$ lines (Fig. 4b), however, pRLK7:Venus-H2B and $p F L S 2$ :Venus-H2B lines had a broader expression pattern than $p H A E$ :Venus-H2B and $p H S L 2$ :Venus-H2B lines, showing fluorescent nuclei in the carpels and the receptacle in addition to the AZs (Fig. 4b).

\section{HSL2 interaction with RLK7 is modulated by the presence of mIDA and PIP1.}

The HAE and HSL2 receptors have been shown to be important for Pseudomonas syringae induced cauline leaf abscission in that hae hsl 2 mutant plants retain their leaves when exposed to the bacterial pathogen ${ }^{19}$. However, during drought induced leaf abscission the presence of a functional HAE, but not HSL2 receptor is necessary; indicating that HAE and HSL2 can diverge in signaling and that HSL2 could be exerting a different function when plants are exposed to stress ${ }^{24}$. We hypothesised that the mIDA dependent induction of defense-associated marker genes could be modulated by other receptor combinations than those regulating organ separation. Based on the recently published sensitized high-throughput interaction assay between extracellular domains (ECDs) of 200 LeucineRich Repeat (LRR) RLKs, we noticed that HAE and HSL2 possess different interaction networks ${ }^{36}$ (Supplementary Fig. 9). Interestingly, LRR-RLKs known to play a function in biotic or abiotic stress responses interacted with HSL2 but not HAE (Supplementary Fig. 9). One of the LRR-RLKs identified as an exclusive unidirectional interactor of HSL2 was RLK7. To further explore the ability of these two receptors to form a complex in planta the HSL2 and RLK7 proteins were expressed as fusions with either a donor (GFP) or an acceptor (mCherry) fluorophore at their C-terminus from an estradiol-inducible $35 \mathrm{~S}$ promoter in N.benthamiana leaves and protein-protein interaction was investigated by the use of Förster Resonance Energy Transfer (FRET). The estradiol inducible promoter was used to achieve comparable expression levels for both receptors in the plasma membrane of leaf epidermal cells (Fig. 5b). We quantified receptor interaction by FRET-Acceptor photobleaching (APB) measurements where the GFP fluorescence after photobleaching of mCherry is calculated as the percentage of change in GFP emission $\left(\mathrm{E}^{\mathrm{FRET}}{ }_{[\%]}\right)$. Only $\mathrm{E}^{\mathrm{FRET}}$ significantly higher than $4 \%$ was regarded to indicate physical interaction between receptor-proteins. As a positive control for optimal energy transfer between donor and acceptor, we fused GFP with mCherry to the C-termini of RLK7 (RLK7-GFP-mCherry) giving an intramolecular $\mathrm{E}^{\mathrm{FRET}}$ of $30 \% \pm 3,71 \%$ (Fig. 5a). RLK7 and HSL2 clearly formed heteromers at the PM independent of which receptor was expressed in fusion with the donor or acceptor fluorophore (HSL2-GFP/RLK7-mCherry, $\mathrm{E}^{\text {FRET }}=15$ $\% \pm 2,49 \%$; RLK7-GFP/HSL2-mCherry, $\mathrm{E}^{\mathrm{FRET}}=10 \% \pm 1,7 \%$ ) (Fig. 5a). These $\mathrm{E}^{\mathrm{FRET}}$ values were significantly different from GFP-only samples of HSL2 (HSL2-GFP, $\mathrm{E}^{\mathrm{FRET}}=-0,9 \% \pm 1,2 \%$ ) and RLK7 (RLK7-GFP, $\mathrm{E}^{\mathrm{FRET}}=0,2 \% \pm 1,7 \%$ ) (Fig. 5a). Importantly, heteromers were formed in the 
absence of exogenous mIDA, and the lack of endogenous mIDA in N.benthamiana leaves ${ }^{12}$ suggests that HSL2 and RLK7 can interact in the absence of mIDA in planta, substantiating the in vitro interaction results (Supplementary Fig. 9). From the sensitized high-throughput interaction assay, RLK7 was not present in the HAE interaction network ${ }^{36}$ (Supplementary Fig. 9) and APB measurements between RLK7 and HAE did not indicate close proximity or interaction between the receptors in planta (RLK7-GFP/HAE-mCherry, $\mathrm{E}^{\mathrm{FRET}}=3,6 \% \pm 1,2 \%$ ) (Fig. 5a).

To explore whether the interaction between RLK7 and HSL2 could be promoted in the presence of mIDA or PIP1 the ECDs of the receptors were expressed as recombinant bait and prey proteins in Drosophila Schneider S2 cells (Supplementary Fig. 10 a,b), and tested in the extracellular interaction assay established previously ${ }^{36}$. Cumulative absorbance (Abs $650 \mathrm{~nm}$ ) for alkaline phosphatase activity measured over $10 \mathrm{~h}$ was significantly higher in the presence of mIDA, but not in the presence of IDA $^{\triangle \mathrm{N} 69}$, indicating enhanced HSL2 ${ }^{\mathrm{ECD}}$ and $\mathrm{RLK} 7^{\mathrm{ECD}}$ interaction by an active mIDA peptide (Fig. 5c). Interestingly, samples treated with PIP1 also augmented the absorbance to the same level as mIDA but no further elevation was seen when combining mIDA and PIP1 (Fig. 5c). Samples treated with unrelated elf18, known to bind and activate the defense related receptor EF-Tu receptor ${ }^{37}$, did not change the absorbance compared to untreated samples (Fig. 5c), showing that the enhanced interaction between RLK7 and HSL2 is peptide specific. HSL2 has been shown to interact with SERK3/BAK1 in an mIDA dependent manner ${ }^{3}$. This was also observed by the extracellular interaction assay, whereas no mIDA mediated interaction was observed between HSL2 and the unrelated PHYTOSULFOKINE RECEPTOR 1 (PSKR1) (Supplementary Fig. 10 and 11 a,b), substantiating the specificity of the mIDA and PIP1 peptides for enhancing RLK $7^{\mathrm{ECD}}$ and HSL2 ${ }^{\mathrm{ECD}}$ interaction.

We next investigated whether RLK7 alone or together with HSL2 could contribute to regulating cell separation during abscission. Phenotypic investigation of floral abscission in an rlk7 single and an rlk7 hsl2 double mutant did not provide any evidence for this (Supplementary Fig. 12), indicating that RLK7 does not have a function in cell wall remodeling. We therefore next investigated the role of RLK7 and HSL2 in regulating defense associated genes.

\section{The immune response activation by IDA is modulated by HSL2 and RLK7}

The same defense-associated marker genes that were induced at the transcriptional levels in response to mIDA after $1 \mathrm{~h}(F R K 1, M Y B 51$, and PEP3) were analyzed by RT-qPCR on tissue from 7 days-old WT, hsl2, rl7, and $r l k 7$ hsl2 mutant seedlings treated with mIDA and compared to untreated seedlings. FRK1 and PEP3 showed significantly higher levels of expression in untreated hsl2 seedlings compared to untreated WT seedlings (Fig. 6 and Supplementary Table 3), indicating that this mutant 
may be associated with an elevated endogenous level of some defense genes. FRK1 expression was significantly reduced in mIDA treated $h s l 2$ and $r l k 7 h s l 2$ mutant seedlings compared to WT seedlings grown in the presence of mIDA, as was the expression level of PEP3 in $r l k 7 h s l 2$ mIDA treated mutant seedlings compared to mIDA treated WT (Fig. 6 and Supplementary Table 3). Unexpectedly, $F R K 1$ and PEP3 expression was higher in $h s l 2$ and $r l k 7$ treated seedlings compared to their untreated mutant counterparts, indicating that the mIDA dependent induction of FRK1 and PEP3 is not only dependent on the presence of RLK7 or HSL2, and that other receptors involved in immunity may be mediating the IDA signal or that the receptors are functionally redundant. This was also apparent from the MYB51 expression that showed increased expression levels in all mIDA treated seedling genotypes tested compared to untreated samples (Fig. 6 and Supplementary Table 3).

Given the lower levels of FRK1 and PEP3 expression in the mIDA treated $r l k 7$ hsl2 mutant seedlings compared to mIDA treated WT seedlings we wanted to investigate whether RLK7 and HSL2 could be signaling in the same cells. A pRLK7:Venus-H2B line was crossed to a $p H S L 2: C e r u l e a n-H 2 B$ expressing line and plants expressing both constructs were inspected for overlapping fluorescent nuclei in 7 days-old roots and AZs from flowers at position 6. In emerging LRs, fluorescent nuclei with overlapping Venus and Cerulean expression were observed in cells surrounding the LRP (Supplementary Fig. 13). At the root tip, the root meristem and lateral root cap had fluorescent nuclei with overlapping expression of the fluorescent tags while in the flowers fluorescent nuclei with overlapping expression were present in some cells of the $\mathrm{AZ}$, carpels and the receptacle (Supplementary Fig. 13). This indicates that RLK7 and HSL2 are present in cells destined for cell separation.

\section{Discussion}

Several plant species can abscise infected organs to limit colonization of pathogenic microorganisms thereby adding an additional layer of defense to the innate immune system ${ }^{18,19}$. It has also been shown that plant pathogens can stimulate the expression of genes encoding peptide ligands suggested, or shown to regulate cell separation and promote abscission ${ }^{11,19}$. However, little is known as to how molecular components regulating abscission can modulate plant defense. In a developmental context, there is an induction of defense associated genes in AZ cells during cell separation and prior to the formation of the suberized layer that functions as a barrier rendering protection to pathogen attacks 1,9,10,16. Here, we address the question of how immune responses triggered by MAMPs and plant signals regulating abscission can cooperate in modulating immunity by activating defense genes in a non-developmental setting in cells undergoing cell separation which are potential entry routes for 
invading pathogens. We explore how the IDA peptide can regulate immune response genes and at the same time be regulated by MAMPs (Fig. 1).

An increase in $\left[\mathrm{Ca}^{2+}\right]_{\text {cyt }}$ and a transient burst of ROS has for a long time been established to form part of MTI ${ }^{32,33}$. The rise in $\left[\mathrm{Ca}^{2+}\right]_{\text {cyt }}$ is important for $\mathrm{RBOH}$ activation both by direct binding to EF hands and by activating Calcium Dependent Kinases which in turn phosphorylate and activate the RBOH enzymes leading to an increase in apoplastic ROS production ${ }^{32}$. ROS can in turn activate $\mathrm{Ca}^{2+}$ influx channels and promote an increase in $\left[\mathrm{Ca}^{2+}\right]_{c y t}$. This mutual regulation between ROS and $\mathrm{Ca}^{2+}$ likely plays a key role in the cell-to-cell long distance propagation of $\mathrm{Ca}^{2+}$ and $\mathrm{ROS}$, and is believed to mediate systemic signaling during biotic and abiotic stresses ${ }^{33}$. mIDA mediates a ROS burst in Arabidopsis rosette leaves (Fig. 2f), also roots expressing the $\mathrm{Ca}^{2+}$ sensor R-GECO1, showed a $\left[\mathrm{Ca}^{2+}\right]_{\mathrm{cyt}}$ wave propagating from the meristematic zone to the root tip in response to mIDA. Moreover, increased $\left[\mathrm{Ca}^{2+}\right]_{\text {cyt }}$ was also observed in response to mIDA during floral organ abscission (Fig. 2a,b,d and e). It is intriguing that mIDA cellular output is similar to that of flg22 ${ }^{32,38}$ yet shows some distinct differences. The spatial distribution of the $\mathrm{Ca}^{2+}$ wave emanated by mIDA differs from that of flg22 (Fig. 2a,b, Supplementary Fig. 3a,b and Movies 1 and 2) and whereas flg22-induced ROS and in part $\mathrm{Ca}^{2+}$ release is dependent on $\mathrm{RBOHD}{ }^{32}$ this does not appear to be the case for mIDA. In contrast to published data, we could not detect ROS accumulation in the hae hsl2 mutant ${ }^{35}$. The cell-type specific pattern of ROS in AZs was unaffected in the rbohd rbohf mutant compared to WT (Fig. 3a), indicating that other NADPH oxidases or cell wall peroxidases expressed in the AZ may be involved in ROS production. This suggests that while IDA and flg22 share many components for their signal transduction, such as the BAK1 co-receptor ${ }^{3,39}$ and MAPK cascade ${ }^{2,28}$, there are other specific components that are likely important to provide differences in signaling.

It has been suggested that the cell type specific accumulation of ROS during abscission is necessary for the correct positioning of a lignin brace restricting cell wall enzymes to the region of organ separation in that rbohd rbohf mutants have been reported to have a defect in floral abscission ${ }^{35}$. The inability of this mutant to undergo cell separation was explained by the diffusion of cell wall digestion enzymes beyond the cells undergoing remodeling ${ }^{35}$. Here we show that precise quantification of abscission phenotypes of several different T-DNA alleles of RBOHD and RBOHF did not reveal any delay in floral abscission (Fig. 3b,c and Supplementary Fig.7). Furthermore, we propose that mIDA induced ROS is associated with an immune response. Supporting this view, ectopic ROS accumulation is seen in AZ cells of the floral abscission mutant nevershed (nev) that has an increased transcription of pathogen response genes ${ }^{10}$. We cannot, however, exclude that mIDA induced $\left[\mathrm{Ca}^{2+}\right]_{\mathrm{cyt}}$ and rises in ROS levels may also have an intrinsic developmental role such as modulating cell wall properties and cell expansion similar to what is observed during FERONIA signaling ${ }^{40,41}$. 
While there is a well of knowledge on the detailed molecular events leading to general immune responses in plants there has been little focus on how plants can mount tissue specific immune responses. We found that flg22 and PIP1 enhance IDA expression by their cognate LRR-RLK immune receptors FLS2 and RLK7, respectively (Fig. 1a,b); and that the promoters of these receptors are active in cells destined for cell separation (Fig. 4a,b and Supplementary Fig. 13). We suggest that, in addition to the defense genes which are developmentally regulated during abscission and that show altered expression in the hae hsl2 mutant ${ }^{9,10,16}$, PAMPs/DAMPs such as flg22 and PIP1 induce IDA expression, which in turn modulates expression of defense-associated genes (Fig. 1c,d). Interestingly, this mIDA dependent induction is in part reliant on the two LRR-RLK receptors RLK7 and HSL2 (Fig. 6). FRK1, an early acting primary defense response gene and a marker gene commonly used to monitor the ability of elicitors to induce defense responses ${ }^{28}$, had significantly lower expression levels in mIDA induced $h s l 2$ and $h s l 2 r l k 7$ mutant seedlings compared to mIDA treated WT seedlings (Fig. 6). Elevated level of expression in un-treated $h s l 2$ seedlings was seen for the FRK1 and PEP3 transcript (Fig. 6), indicating that the $h s l 2$ mutant itself has an activated immune response. Unlike the significant decrease in expression seen for $P E P 3$ in mIDA treated $h s l 2$ rlk7 mutant seedlings compared to treated WT seedlings, expression of MYB51 showed no difference in expression levels for any of the treated mutant genotypes compared to treated WT (Fig. 6). Interestingly, MYB51 is downregulated in the $\mathrm{AZ}$ of hae hsl2 compared to $\mathrm{WT}^{9}$, indicating that the increase in expression seen in mIDA treated seedlings is dependent on the presence of HAE and HSL2 and not HSL2 and RLK7. The significant increase in expression of FRK1 and PEP3 in mIDA treated $r l k 7$ samples, and increased PEP3 expression in treated hsl2 samples compared to untreated samples of the same genotype shows that other receptors may play a role in this regulation. In the nev mutant that has an over-activation of SERK1 signaling there was a 3,7 and 4,1 fold increase in expression of $F R K 1$ and PEP3 compared to WT, indicating that SERK signaling could be important ${ }^{10,42}$.

We propose a model (Fig. 7) where IDA-mediated signaling can take place through different receptor complexes to coordinate developmental abscission and defense. We suggest that biotic and abiotic elicitors enhance IDA expression in cells that have the potential to undergo cell separation. The heteromerisation between HSL2 and RLK7 (Fig. 5 a) is promoted upon IDA and PIP1 perception (Fig. 5c) and leads to the activation of a set of defense-associated genes. PIP1 could thus function as a molecular switch recruiting HSL2 together with IDA into a signaling module with RLK7 to promote immune responses. In the event that an organ, such as a leaf, is challenged by a pathogen, the activation of defense-associated genes in cells at the joint between the organ and the plant could act as a protective barrier. In cells that undergo cell separation, without the loss of an organ, induction of defense genes would play a similar function. Indeed both HSL2 and RLK7 are expressed in root cap 
cells that undergo sloughing (Supplementary Fig. 13). During cell separation RLK7 signaling would add an additional layer of protection to the defense genes that are developmentally induced, since RLK7 and PIP1 do not appear to play a role in regulating cell separation (Supplementary Fig. 12 and $\left.{ }^{23}\right)$.

Phylogenetic analyses suggest that HSL2 was present in species that gave rise to the angiosperms and that $H A E$ arose later in evolution ${ }^{43}$. It is therefore possible that IDA and HSL2 originally had a function in plant innate immunity and later developed a specified role in regulating cell separation yet keeping the ability to respond to environmental stresses. The similarity between PIP/PIPL and IDA/IDL peptides would support this, in that the PIP and PIPL peptides are involved in regulating stress responses ${ }^{11,23}$. A common function for these family of peptides is also apparent from their induction by various abiotic stresses like salt ${ }^{11}$ (and Genevestigator Experiment ID: AT- 00120). Given the similarities in signaling components for pathways mediating cell separation and defense, it will be exciting to further explore the tight interplay between these processes.

\section{Materials and methods}

Accession numbers of genes studied in this work: HSL2 At5g65710, RLK7 At1g09970, IDA At1g68765, FLS2 At5g46330, HAE At4g28490, MYB51 At1g18570, PIP1 At3g61430, RBOHD At5g47910, RBOHF At1g64060, PEP3 At5g64905, WRKY33 At2g38470, FRK1 At2g19190. Plant lines used in this work: Ecotype Colombia-0 (Col-0) was used as wild type (WT). Mutant line: hae (SALK 021905), hsl2, (SALK 030520), rlk7 (SALK_094492), fls2 (SALK_062054), rbohd (SALK_070610- exon 7, SALK_120299-exon 7, CS9555 [At rbohd-D3] ${ }^{44}$ exon 5), rbohf (SALK_059888-intron 3, SALK_057041-intron 4, CS68748 [At rbohf-F3] exon $1^{44}$ ).

SALK lines were provided from Nottingham Arabidopsis Stock Centre (NASC).

\section{Plant lines}

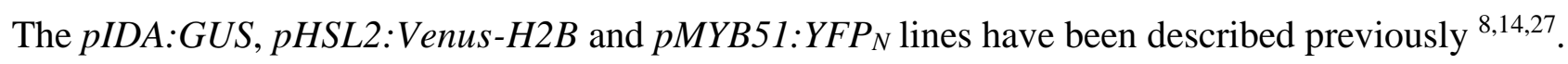
The promoters of HAE (1601 bp ${ }^{8}$ ) and HSL2 (2300 bp ${ }^{43}$ ) were available in the pDONRZeo vector (Thermo Fischer Scientific). Sequences corresponding to the RLK7 (1880 bp ${ }^{45}$ ) and FLS2 promoters (988 bp ${ }^{46}$ ) were amplified from WT DNA (primers are listed in table 2) and cloned into the pDONRZeo vector (Thermo Fischer Scientific). All promoter constructs were further recombined into the promotor:Venus (YFP)-H2B and Cerulean(CFP)-H2B destination vectors ${ }^{47}$ using the Invitrogen Gateway cloning system (Thermo Fischer Scientific). Constructs were transformed into Agrobacterium tumefacience (A.tumefacience) C58 and the floral dip method ${ }^{48}$ was used to generate transgenic lines. Single-copy homozygous plant lines were selected and used in this study. Full- 
length CDS of HAE, HSL2 and RLK7 were cloned in frame with expression vectors containing an estradiol inducible promotor and a C-terminal fluorescent molecule of GFP, mCherry or GFPmCherry ${ }^{49}$ using the Invitrogen Gateway cloning system. Plasmids were transformed into A.tumefacience C58 and further used for transient expression of receptor proteins in Nicotiana benthamiana (N.benthamiana) leaves. The CDS of HAE was cloned into the pEarleyGate101 destination vector 50 using the Invitrogen Gateway cloning system and transformed into A.tumefacience C58 and further used to generate the 35S:HAE:YFP lines.

\section{Growth conditions}

Plants were grown in long day conditions ( $8 \mathrm{~h}$ dark and $16 \mathrm{~h}$ light) at $22^{\circ} \mathrm{C}$. Seeds were surface sterilized and plated out on MS-2 plates, stratified for $24 \mathrm{~h}$ at $4{ }^{\circ} \mathrm{C}$ and grown on plates for 7 days before transferred to soil.

\section{Peptide sequences}

Peptides used in this study were ordered from BIOMATIK. Peptide sequences are listed in Supplementary Table 1 .

\section{Primers}

Primers for genotyping and generation of constructs were generated using VectorNT1. Gene specific primers for RT-qPCR were generated using Roche Probe Library Primer Design. All primers are listed in Supplementary Table 2.

\section{Histochemical GUS assay}

Seven days-old seedlings were pre-incubated for $12 \mathrm{~h}$ in liquid MS-2 medium containing stimuli of interest; $1 \mu \mathrm{M}$ peptide (table 1), 60 mM Mannitol (M4125 - Sigma), $20 \mu \mathrm{g} / \mathrm{mL}$ Chitin (C9752 Sigma), $50 \mathrm{mM} \mathrm{NaCl}$ and then stained for GUS activity following the protocol previously described

5 . Roots were pictured using a Zeiss Axioplan2 microscope with an AxioCam HRc, 20x air objective. Seedlings The assay was performed on 10 individual roots and the experiment was repeated 3 times.

\section{Fluorescent GUS assay}

Seven days-old seedlings were pre-incubated for $12 \mathrm{~h}$ in liquid MS-2 medium with or without stimuli of interest; $1 \mu \mathrm{M}$ peptide (table 1), $60 \mathrm{mM}$ Mannitol (M4125 - Sigma), $20 \mu \mathrm{g} / \mathrm{mL}$ Chitin (C9752 Sigma), $50 \mathrm{mM} \mathrm{NaCl}$. After treatment, 10 seedlings were incubated in wells containing $1 \mathrm{~mL}$ reaction mix described in ${ }^{20}$ conatining: 10 mM EDTA (pH 8.0), 0,1\% SDS, 50 mM Sodium Phosphate (pH 
7.0), 0,1\% Triton X-100, $1 \mathrm{mM}$ 4-MUG (, M9130-Sigma) and incubated at $37^{\circ} \mathrm{C}$ for $6 \mathrm{~h}$. Six $100 \mu \mathrm{l}$ aliquots from each well were transferred to individual wells in a microtiter plate and the reaction was stopped by adding $50 \mu \mathrm{l}$ of stop reagent (1 M Sodium Carbonate) to each well. Fluorescence was detected by the use of a Wallac 1420 VICTOR2 microplate luminometer (PerkinElmer) using an excitation wavelength of $365 \mathrm{~nm}$ and a filter wavelength of $430 \mathrm{~nm}$. Each experiment was repeated 3 times.

\section{Confocal laser microscopy of roots and flowers expressing promoter:Venus/YFP-H2B and promoter: Cerulean-H2B constructs}

Imaging of 7 days-old roots was performed on a LSM 880 Airyscan confocal microscope equipped with two flanking PMTs and a central 32 array GaAsP detector. Images were acquired with Zeiss Plan-Apochromat 20x/0.8 WD=0.55 M27 objective and excited with lasers light of $405 \mathrm{~nm}, 488 \mathrm{~nm}$ and $561 \mathrm{~nm}$. Roots were stained by $1 \mu \mathrm{M}$ propidium iodide for $10 \mathrm{~min}$ and washed in $\mathrm{dH}_{2} \mathrm{O}$ before imaging. Imaging of flowers was performed on an Andor Dragonfly spinning disk confocal using an EMCCD iXon Ultra detector. Images were acquired with a 10x plan Apo NA 0,45 dry objective and excited with laser light of $405 \mathrm{~nm}, 488 \mathrm{~nm}$ and $561 \mathrm{~nm}$. Maximum intensity projections of z-stacks s were acquired with step size of $1,47 \mu \mathrm{m}$. Image processing was performed in FIJI ${ }^{51}$. These steps are: background subtraction, gaussian blur/smooth, brightness/contrast. Imaging was performed at the NorMIC Imaging platform.

\section{Protein-protein interaction by FRET-acceptor PhotoBleaching (FRET-APB)}

FRET-APB was performed on N.benthamiana leaves transiently expressing full-length receptors as described previously ${ }^{12}$. FRET-APB values was measured as previously described ${ }^{49}$ using a Zeiss LSM880 Airyscan. A Plan-Apochromat 20x/0.8 WD=0.55 M27 objective, an optical zoom of 5 x, frame size of $256 \times 256$ pixels and scan speed of $629 \mathrm{msec}$ per frame were used for all measurements. Frame size, laser-power and gain were kept constantly throughout all measurements. $\mathrm{E}^{\mathrm{FRET}}$ was measured via GFP fluorescence intensity increase after photobleaching of the acceptor mCherry using the Zeiss FRET measurement option $\left(\mathrm{E}^{\mathrm{FRET}}=\left(\mathrm{GFP}_{\mathrm{after}}-\mathrm{GFP}_{\text {before }}\right) / \mathrm{GFP}_{\text {after }} \times 100\right)$. Only measurements with minimal fluctuations of GFP and mCherry were used. $\mathrm{E}^{\mathrm{FRET}}$ significantly higher than $4 \%$ was regarded to indicate physical interaction between receptor-proteins as described in ${ }^{49}$. All measurements were performed 15 times and each experiment was repeated 3 times. The donoronly sample (GFP) was used as a negative control. FRET-APB measurements were performed at the NorMIC Imaging platform. 


\section{Molecular cloning of LRR-RLK extracellular domains}

This step was performed as described previously in ${ }^{36}$.

\section{Secreted expression of LRR-RLK extracellular domains}

The extracellular domains (ECDs) of HSL2, RLK7, BAK1, and PSKR1 (nucleotide and amino acid sequences are listed in Supplementary Table 4) cloned into the pECIA2 (for expression as a bait) and pECIA14 (for expression as a prey) vectors were expressed using transient transfection of Drosophila S2 cells cultured at $27^{\circ} \mathrm{C}$. Upon transfection using ExpreS ${ }^{2} \mathrm{TR}$ (ExpreS ${ }^{2}$ ion Biotechnologies), the culturing temperature was changed to $25^{\circ} \mathrm{C}$. Twenty-four $\mathrm{h}$ after transfection, protein expression was induced with $1 \mathrm{mM} \mathrm{CuSO}_{4}$ and supernatant was collected four days after induction. Protease inhibitors (Sigma) and $0.02 \% \mathrm{NaN} 3$ were added to the medium (ESF 921, Expression Systems) containing the recombinant ECDs and then stored at $4{ }^{\circ} \mathrm{C}$ before use. The cell supernatant was assessed for recombinant protein expression by western blotting using anti-V5 antibodies (Invitrogen) for the baits and anti-FLAG antibodies for the preys.

\section{Targeted interaction assay using CSI method}

The interaction assays were performed as detailed previously ${ }^{36}$ with the slight modifications indicated below. Schneider's medium containing recombinant bait ECD of HSL2 and prey ECDs of RLK7, BAK1, and PSKR1 (protein sequences are listed in Supplementary Table 4) were mixed together and diluted in a PBS buffer containing $1 \mathrm{mM} \mathrm{CaCl}_{2}, 1 \mathrm{mM} \mathrm{MgCl}$ (equilibration buffer) and $0.1 \%$ bovine serum albumin (BSA; Sigma) to obtain a final eightfold dilution in a volume of $100 \mu 1$. Each bait-prey combination was incubated for $2 \mathrm{~h}$ at $4{ }^{\circ} \mathrm{C}$ in the presence and absence of peptides. Meanwhile, the Protein-A-coated plates were rehydrated by 3 consecutive washes in a PBS solution containing $0.1 \%$ Tween-20 before use. The bait- prey interaction combinations were captured directly on the 96-well protein-A-coated plates (Thermo Fisher Scientific) by overnight incubation at $4{ }^{\circ} \mathrm{C}$. The excess of bait-prey mixtures was washed away using equilibration buffer which had previously been added the alkaline phosphatase substrate (KPL 50-88-02) the same peptide concentration was kept during the washing steps. Upon addition of the substrate, alkaline phosphatase activity was monitored for $10 \mathrm{~h}$ at room temperature by measuring the absorbance at $650 \mathrm{~nm}$ using a Synergy H4 Multi-Mode plate reader (BioTek). Images of the 96-well plates were acquired for visual inspection. The complete set of raw absorbance values was combined and then subjected to post experimental calculations and statistical analysis. 


\section{Calcium imaging using the R-GECO1 sensor}

$\left[\mathrm{Ca}^{2+}\right]_{\mathrm{cyt}}$ in roots were detected using WT plants expressing the cytosolic localized single-fluorophore based $\mathrm{Ca}^{2+}$ sensor, R-GECO1 ${ }^{52}$. Measurements were performed using a confocal laser scanning microscopy Leica TCS SP8 STED 3X using a 20x multi NA 0.75 objective. R-GECO1 was excited with a white light laser at $561 \mathrm{~nm}$ and its emission was detected at $590 \mathrm{~nm}$ to $670 \mathrm{~nm}$ using a HyD detector. Laser power and gain settings were chosen for each experiment to maintain comparable intensity values. Images were recorded with a frame rate of 5 seconds at $400 \mathrm{~Hz}$. Sample mounting was performed as described in ${ }^{53}$. Seedlings were incubated overnight in half strength $\mathrm{MS}, 21^{\circ} \mathrm{C}$ and continuous light conditions before the day of imaging. For mIDA and flg22 two-fold concentrations were prepared in half strength MS. mIDA or flg22 were added in a 1:1 volume ratio to the imaging chamber (final concentration $1 \mu \mathrm{M}$ ). ATP was prepared in a 100-fold concentration in half strength MS and added as a last treatment in a 1:100 volume ratio (final concentration $1 \mathrm{mM}$ ) to the imaging chamber as a positive control for activity of the R-GECO1 sensor (Movie 3). Image processing was performed in FIJI ${ }^{51}$. These steps are: background subtraction, gaussian blur, MultiStackReg v1.45 (http://bradbusse.net/sciencedownloads.html), 32-bit conversion, threshold. Royal was used as a look up table. Fluorescence intensities of indicated ROIs were obtained from the 32-bit images ${ }^{53}$. Normalization was done using the following formula $\Delta F / F=\left(F-F_{0}\right) / F_{0}$ where $F_{0}$ represents the mean of at least $1 \mathrm{~min}$ measurement without any treatment. R-GECO1 measurements were performed at the Center for Advanced imaging (CAi) at HHU.

\section{Calcium measurements using the Aequorin (pMAQ2) sensor}

$\left[\mathrm{Ca}^{2+}\right]_{\text {cyt }}$ in seedlings and flowers were detected using WT plants expressing p35S-apoaequorin (pMAQ2) located to cytosol (Aeq) ${ }^{55}$. Aequorin luminescence was measured as previously described 56. Emitted light was detected by the use of a Wallac 1420 VICTOR2 microplate luminometer (PerkinElmer). Differences in Aeq expression levels due to seedling size and expression of sensor were corrected by using luminescence at specific time point $(\mathrm{L}) / \mathrm{Max}$ Luminescence $\left(\mathrm{L}_{\max }\right)$. $\mathrm{L}_{\max }$ was measured after peptide treatment by individually adding $100 \mu \mathrm{L} 2 \mathrm{M} \mathrm{CaCl}_{2}$ to each well and measuring luminescence constantly for 180 seconds ${ }^{57} .2 \mathrm{M} \mathrm{CaCl}_{2}$ disrupts the cells and releases the Aeq sensor into the solution where it will react with $\mathrm{Ca}^{2+}$ and release the total possible response in the sample $\left(\mathrm{L}_{\max }\right)$ in form of a luminescent peak. A final concentration of $1 \mu \mathrm{M}$ mIDA was added to each wells at the start of measurements. For seedlings 3 independent experiments were performed with 12 replications in each experiment. For flowers 3 independent experiments were performed with 4-6 replications in each experiment. 


\section{Measurements of reactive oxygen species (ROS)}

ROS production was monitored by the use of a luminol-dependent assay as previously described ${ }^{12}$ using a Wallac 1420 VICTOR2 microplate luminometer (PerkinElmer). Arabidopsis leaves expressing 35S:HAE:YFP were cut into leaf discs and incubated in water overnight before measurements. A final concentration of $1 \mu \mathrm{M}$ mIDA was added to each well at the start of measurements. All measurements were performed on 6 leaf discs and each experiment was repeated 3 times.

\section{ROS stain (H2DCF-DA)}

Flowers at position 6 were gently incubated in staining solution $\left(25 \mu \mathrm{M} \quad\left(2^{\prime}, 7^{\prime}-\right.\right.$ dichlorodihydrofluorescein diacetate) ( $\mathrm{H}_{2}$ DCF-DA) (Sigma-Aldrich, D6883), $50 \mathrm{mM} \mathrm{KCL}, 10 \mathrm{mM}$ MES) for $10 \mathrm{~min}$ and further washed 3 times in wash solution (50 mM KCL, $10 \mathrm{mM} \mathrm{MES})$. For the hae hsl2 mutant the floral organs were forcibly removed immediately before imaging. Imaging was done using a Dragonfly Airy scan spinning disk confocal microscope, excited by a $488 \mathrm{~nm}$ laser. A total of 9 flowers per genotype were imaged. The experiment was repeated 2 independent times.

\section{Real time quantitative PCR (RT-qPCR)}

Seven days-old Arabidopsis seedlings (WT, $r l k 7, h s l 2$ and $h s l 2$ rlk7) grown vertically on $1 / 2$ sucrose MS-2 plates were transferred to liquid 1/2 MS-2 medium (non-treated) and liquid 1/2 MS-2 medium containing $1 \mu \mathrm{M}$ mIDA and incubated in growth chambers for $1 \mathrm{~h}$ or $12 \mathrm{~h}$. Seedlings were flashfrozen in liquid nitrogen before total RNA was extracted using Spectrum ${ }^{\mathrm{TM}}$ Plant Total RNA Kit (SIGMA Aldrich). cDNA synthesis was performed as previously described ${ }^{58}$. RT-qPCR was performed according to protocols provided by the manufacturer using FastStart Essential DNA Green Master (Roche) and LightCycler96 (Roche) instrument. ACTIN2 was used to normalize mRNA levels as described in ${ }^{58}$. Three biological replicates and 4 technical replicates including standard curves were performed for each sample.

\section{Petal Break strength (pBS)}

The force required to remove a petal at a given position on the inflorescence was measured in gram equivalents using a load transducer as previously described ${ }^{5}$. Plants were grown until they had at least 15 positions on the inflorescence. A minimum of 15 petals per position were measured. pBS measurements were performed on WT, rbohd rbohf (SALK_070610 SALK_059888) and hae hsl2 (SALK_021905 SALK_030520) plants. 


\section{Statistical methods}

Two tailed students t-test $(\mathrm{p}<0.05)$ was used to identify significant differences in the fluorescent GUS assay by comparing treated samples to untreated samples of the same plant line. Two tailed students t-test $(\mathrm{p}<0.05)$ was used to identify significant differences in RT-qPCR analysis by comparing all untreated samples to mIDA treated samples of same genotype $(*)$, by comparing all untreated genotypes to untreated WT (**) and by comparing all mIDA treated genotypes to mIDA treated WT $(* * *)$ (statistical data presented in Supplementary table 3). Two tailed students t-test with $(\mathrm{p}<0.05)$ was used in the petal break strength (pBS) measurements to identify significant differences from WT at a given position on the inflorescence. Statistical significance of each HSL2 ${ }^{\mathrm{ECD}}$ (bait) and RLK $7^{\mathrm{ECD}}, \mathrm{BAK} 1^{\mathrm{ECD}}$, PSKR $1^{\mathrm{ECD}}$ (preys) interactions in the presence of peptides (mIDA, PIP1, IDA $^{\Delta N 69}$, and elf18) in comparison to interaction in the absence of peptide (-) was determined using a One-Way ANOVA with pairwise multiple comparisons and the resulting P values were corrected for multiple testing using the Holm method ${ }^{36}$ ( $\left.p<0.0001\right)$.

\section{Acknowledgements}

We thank M.K. Anker, I.M. Stø, V. Iversen and R. Falleth for technical assistance in the laboratory and phytotrone. We thank the NorMic Imaging platform for the use and technical support. We would like to extend a thank-you and a welcome to Alex Smakowska for placing the finishing touch on this manuscript, and T. Ullerud and I. De Smet for insightful discussions. This work was supported by the Research Council of Norway (grant 230849) to V.O. and M.A.B. By the Hertha Firnberg Programme post-doctoral fellowship (T-947) from the FWF Austrian Science Fund, Vienna, Austria.to E.S-L. Work by R. Simon and and M.B. was supported through CEPLAS.

\section{Author contributions}

V.O. generated Arabidopsis lines and constructs, tested IDA expression to biotic and abiotic stress, performed gene expression studies, phenotypic analysis of mutants, ROS measurements and FRET experiments. V.O. and M.B. performed $\mathrm{Ca}^{2+}$ measurements. E.S-L. and R. Schneeweiss performed the extracellular interaction assay. P.M. performed IDA expression studies. V.O., E.S-L., M.B., P.M., Y.B., R.Simon and M.A.B designed experiments, analyzed data, and drafted the manuscript. V.O. and M.A.B wrote the paper with input from all authors. 


\section{References}

1 Roberts, J. A., Whitelaw, C. A., Gonzalez-Carranza, Z. H. \& McManus, M. T. Cell separation processes in plants- models, mechanisms and manipulation. Ann Bot-London 86, 223-235 (2000).

2 Cho, S. K. et al. Regulation of floral organ abscission in Arabidopsis thaliana. Proc Natl Acad Sci USA 105, 15629-15634 (2008).

3 Meng, X. et al. Ligand-induced receptor-like kinase complex regulates floral organ abscission in Arabidopsis. Cell reports 14, 1330-1338 (2016).

4 Santiago, J. et al. Mechanistic insight into a peptide hormone signaling complex mediating floral organ abscission. Elife 5, e15075 (2016).

5 Stenvik, G. E. et al. The EPIP peptide of INFLORESCENCE DEFICIENT IN ABSCISSION is sufficient to induce abscission in Arabidopsis through the receptorlike kinases HAESA and HAESA-LIKE2. Plant Cell 20, 1805-1817 (2008).

6 Shi, C.-L. et al. Arabidopsis class I KNOTTED-Like Homeobox Proteins act downstream in the IDA-HAE/HSL2 floral abscission signaling pathway. Plant Cell 23, 2553-2567 (2011).

7 Butenko, M. A. et al. INFLORESCENCE DEFICIENT IN ABSCISSION controls floral organ abscission in Arabidopsis and identifies a novel family of putative ligands in plants. Plant Cell 15, 2296-2307 (2003).

8 Kumpf, R. P. et al. Floral organ abscission peptide IDA and its HAE/HSL2 receptors control cell separation during lateral root emergence. Proc Natl Acad Sci U S A 110, 5235-5240, (2013).

9 Niederhuth, C., Patharkar, O. R. \& Walker, J. Transcriptional profiling of the Arabidopsis abscission mutant hae hsl2 by RNA-Seq. BMC Genomics 14, 37 (2013).

10 Taylor, I. \& Walker, J. C. Transcriptomic evidence for distinct mechanisms underlying abscission deficiency in the Arabidopsis mutants haesalhaesa-like 2 and nevershed. BMC Res Notes 11, 754 (2018).

11 Vie, A. K. et al. The IDA/IDA-LIKE and PIP/PIP-LIKE gene families in Arabidopsis: phylogenetic relationship, expression patterns, and transcriptional effect of the PIPL3 peptide. J exp bot. 66, 5351-5365 (2015).

12 Butenko, M. A. et al. Tools and strategies to match peptide-ligand receptor pairs. Plant cell 26, 1838-1847 (2014). 
13 Schardon, K. et al. Precursor processing for plant peptide hormone maturation by subtilisin-like serine proteinases. Science 14, 1594-1597 (2016).

14 Shi, C.-L. et al. The dynamics of root cap sloughing in Arabidopsis is regulated by peptide signalling. Nat Plants 4, 596-604 (2018).

15 Agustí, J., Merelo, P., Cercós, M., Tadeo, F. R. \& Talón, M. Comparative transcriptional survey between laser-microdissected cells from laminar abscission zone and petiolar cortical tissue during ethylene-promoted abscission in citrus leaves. BMC Plant Biol. 9, 127 (2009).

16 Cai, S. \& Lashbrook, C. C. Stamen abscission zone transcriptome profiling reveals new candidates for abscission control: enhanced retention of floral organs in transgenic plants overexpressing Arabidopsis ZINC FINGER PROTEIN2. Plant Physiol. 146, 1305-1321 (2008).

17 Wang, X. et al. IDL6-HAE/HSL2 impacts pectin degradation and resistance to Pseudomonas syringae pv tomato DC3000 in Arabidopsis leaves. Plant J. 89, 250-263 (2017).

18 Kissoudis, C. et al. Responses to combined abiotic and biotic stress in tomato are governed by stress intensity and resistance mechanism. J exp bot. 67, 5119-5132 (2016).

19 Patharkar, O. R., Gassmann, W. \& Walker, J. C. Leaf shedding as an anti-bacterial defense in Arabidopsis cauline leaves. PLoS genetics 13, e1007132 (2017).

20 Blázquez, M. Quantitative GUS Activity assay in intact plant tissue. CSH Protoc. 2007, pdb.prot4688 (2007).

21 Chinchilla, D., Bauer, Z., Regenass, M., Boller, T. \& Felix, G. The Arabidopsis receptor kinase FLS2 binds flg22 and determines the specificity of flagellin perception. Plant Cell 18, 465-476 (2006).

22 Gómez-Gómez, L. \& Boller, T. FLS2: An LRR Receptor-like kinase involved in the perception of the bacterial elicitor flagellin in Arabidopsis. Mol Cell 5, 1003-1011 (2000).

23 Hou, S. et al. The secreted peptide PIP1 amplifies immunity through Receptor-Like Kinase 7. PLOS Pathog. 10, e1004331 (2014).

24 Patharkar, O. R. \& Walker, J. C. Core mechanisms regulating developmentally timed and environmentally triggered Abscission. Plant physiol. 172, 510-520 (2016).

25 Birkenbihl, R. P., Kracher, B., Roccaro, M. \& Somssich, I. E. Induced genome-wide binding of three Arabidopsis WRKY transcription factors during early MAMPtriggered immunity. Plant Cell 29, 20-38 (2017). 
26 Frerigmann, H. et al. Regulation of pathogen-triggered tryptophan metabolism in Arabidopsis thaliana by MYB transcription factors and indole glucosinolate conversion products. Mol. Plant 9, 682-695 (2016).

27 Poncini, L. et al. In roots of Arabidopsis thaliana, the damage-associated molecular pattern AtPep1 is a stronger elicitor of immune signalling than flg22 or the chitin heptamer. PloS one 12, e0185808 (2017).

28 Asai, T. et al. MAP kinase signalling cascade in Arabidopsis innate immunity. Nature 415, 977-983 (2002).

29 He, P. et al. Specific bacterial suppressors of MAMP signaling upstream of MAPKKK in Arabidopsis innate immunity. Cell 125, 563-575 (2006).

30 Huffaker, A., Pearce, G. \& Ryan, C. A. An endogenous peptide signal in Arabidopsis activates components of the innate immune response. Proc Natl Acad Sci U S A 103, 10098-10103 (2006).

31 Birkenbihl, R. P., Diezel, C. \& Somssich, I. E. Arabidopsis WRKY33 is a key transcriptional regulator of hormonal and metabolic responses toward Botrytis cinerea infection. Plant physiol. 159, 266-285 (2012).

32 Kadota, Y., Shirasu, K. \& Zipfel, C. Regulation of the NADPH oxidase RBOHD during plant immunity. Plant Cell Physiol 56, 1472-1480 (2015).

33 Steinhorst, L. \& Kudla, J. Calcium and reactive oxygen species rule the waves of signaling. Plant Physiol. 163, 471-485 (2013).

34 Patharkar, O. R. \& Walker, J. C. Floral organ abscission is regulated by a positive feedback loop. Proc Natl Acad Sci U S A 112, 2906-2911 (2015).

35 Lee, Y. et al. A Lignin Molecular Brace Controls Precision Processing of Cell Walls Critical for Surface Integrity in Arabidopsis. Cell 173, 1468-1480 (2018).

36 Smakowska-Luzan, E. et al. An extracellular network of Arabidopsis leucine-rich repeat receptor kinases. Nature 553, 342 (2018).

37 Zipfel, C. et al. Perception of the bacterial PAMP EF-Tu by the receptor EFR restricts Agrobacterium-mediated transformation. Cell 125, 749-760 (2006).

38 Kadota, Y. et al. Direct regulation of the NADPH oxidase RBOHD by the PRRassociated kinase BIK1 during plant immunity. Mol. Cell 54, 43-55 (2014).

39 Chinchilla, D. et al. A flagellin-induced complex of the receptor FLS2 and BAK1 initiates plant defence. Nature 448, 497 (2007). 
40 Dünser, K. et al. Extracellular matrix sensing by FERONIA and Leucine-Rich Repeat Extensins controls vacuolar expansion during cellular elongation in Arabidopsis thaliana. EMBO J. 38, e100353 (2019).

41 Feng, W. et al. The FERONIA receptor kinase maintains cell-wall integrity during salt stress through $\mathrm{Ca}^{2+}$ ) Signaling. Curr Bio.l 28, 666-675 (2018).

42 Taylor, I., Baer, J., Calcutt, R. \& Walker, J. C. Hypermorphic SERK1 mutations function via a SOBIRl pathway to activate floral abscission signaling. Plant Physiol. 180, 12191229 (2019).

43 Stø, I. M. et al. Conservation of the abscission signaling peptide IDA during Angiosperm evolution: withstanding genome duplications and gain and loss of the receptors HAE/HSL2. Frontiers Plant Sci. 6, 931 (2015).

44 Torres, M. A., Dangl, J. L., Jones, J.D., Arabidopsis gp91phox homologues AtrbohD and AtrbohF are required for accumulation of reactive oxygen intermediates in the plant defense response. Proc Natl Acad Sci U S A 99 517-522 (2002).

45 Pitorre, D. et al. RLK7, a leucine-rich repeat receptor-like kinase, is required for proper germination speed and tolerance to oxidative stress in Arabidopsis thaliana. Planta 232, 1339-1353 (2010).

46 Robatzek, S., Chinchilla, D. \& Boller, T. Ligand-induced endocytosis of the pattern recognition receptor FLS2 in Arabidopsis. Gene Dev. 20, 537-542 (2006).

47 Somssich, M., Bleckmann, A. \& Simon, R. Shared and distinct functions of the pseudokinase CORYNE (CRN) in shoot and root stem cell maintenance of Arabidopsis. J Exp Bot. 67, 4901-4915 (2016).

48 Clough, S. J. \& Bent, A. F. Floral dip: A simplified method for Agrobacterium-mediated transformation of Arabidopsis thaliana. Plant J. 16, 735-743 (1998).

49 Bleckmann, A., Weidtkamp-Peters, S., Seidel, C. A. \& Simon, R. Stem cell signaling in Arabidopsis requires CRN to localize CLV2 to the plasma membrane. Plant Physiol. 152, 166-176 (2010).

50 Earley, K. W. et al. Gateway-compatible vectors for plant functional genomics and proteomics. Plant J. 45, 616-629 (2006).

51 Schindelin, J. et al. Fiji: an open-source platform for biological-image analysis. Nat. Methods 9, 676 (2012).

52 Keinath, N. F. et al. Live cell imaging with R-GECO1 sheds light on flg22- and Chitininduced transient $\left[\mathrm{Ca}^{2+}\right]$ cyt patterns in Arabidopsis. Mol. Plant 8, 1188-1200 (2015). 
53 Waadt, R., Krebs, M., Kudla, J., Schumacher, K. Multiparameter imaging of calcium and abscisic acid and high-resolution quantitative calcium measurements using RGECO1-mTurquoise in Arabidopsis. New Phytol. 216, 303-320 (2017).

54 Krebs, M. \& Schumacher, K. Live cell imaging of cytoplasmic and nuclear $\mathrm{Ca}^{2+}$ dynamics in Arabidopsis roots. CSH Protoc. 8, 776-780 (2013).

55 Knight, M. R., Campbell, A. K., Smith, S. M. \& Trewavas, A. J. Transgenic plant aequorin reports the effects of touch and cold-shock and elicitors on cytoplasmic calcium. Nature 352, 524-526 (1991).

56 Ranf, S. et al. Defense-related calcium signaling mutants uncovered via a quantitative high-throughput screen in Arabidopsis thaliana. Mol. Plant 5, 115-130 (2012).

57 Ranf, S. et al. A lectin S-domain receptor kinase mediates lipopolysaccharide sensing in Arabidopsis thaliana. Nat. Immunol. 16, 426-433 (2015).

58 Grini, P. E. et al. The ASH1 HOMOLOG 2 (ASHH2) histone H3 methyltransferase is required for ovule and anther development in Arabidopsis. PLoS One 4, e7817 (2009).

\section{Figure Legends}

\section{Fig. 1: IDA is induced by biotic stress and triggers defense-associated marker genes}

a, Enhanced pIDA:GUS expression after 12 hours (h) treatment with $1 \mu \mathrm{M}$ flg22 or $1 \mu \mathrm{M}$ PIP1 is observed in cells surrounding emerging lateral roots. Controls were either not subjected to any stimuli (No treatment) or exposed to $1 \mu \mathrm{M}$ of the inactive IDA peptide, IDA ${ }^{\Delta \mathrm{N} 694,12}$. No enhanced flg22 and PIP1 induced expression is observed in pIDA:GUS fls2 or pIDA:GUS rlk7 plants compared to plants grown without the presence of any stimuli (No treatment). Representative picture of $\mathrm{n}=10$, experiment repeated 3 times, scale bar $=50 \mu \mathrm{m}$. b. Normalized emitted fluorescence of fluorochrome 4-methyl umbelliferone (4-MU) in 7 days-old seedlings after $12 \mathrm{~h}$ treatment with $1 \mu \mathrm{M}$ PIP1 and 1 $\mu \mathrm{M}$ flg22 in pIDA:GUS seedlings, pIDA:GUS fls2 seedlings and pIDA:GUS rlk7 seedlings. Controls were either not subjected to any stimuli (No treatment) or exposed to $1 \mu \mathrm{M} \mathrm{IDA}^{\Delta \mathrm{N} 69} \cdot \mathrm{n}=6$, experiment repeated 3 times. ${ }^{*}=$ significantly different from the non-treated (No treatment) sample $(\mathrm{p}<0.05$, student t-test, two tailed). c, $p M Y B 51: Y F P_{N}$ expression is enhanced in roots after $7 \mathrm{~h}$ exposure to 1 $\mu \mathrm{M}$ mIDA peptide compared to untreated roots (control), scale bar $=50 \mu \mathrm{m}$, maximum intensity projections of z-stacks, magenta $=$ propidium iodide stain. d, Transcripts of FRK1, MYB51, PEP3 and WRKY33 in WT seedlings exposed to $1 \mu \mathrm{M}$ mIDA for $1 \mathrm{~h}$ (light gray) and to $1 \mu \mathrm{M}$ mIDA for 12 h (dark gray) compared to untreated tissue (black). RNA levels were measured by RT-qPCR analysis. ACTIN was used to normalize mRNA levels. $\mathrm{n}=4$, three biological replicates. $*=$ significantly 
different from untreated sample ( $\mathrm{p}<0.05$, student t-test, two tailed). Statistical analyses are presented in Supplementary Table 3.

\section{Fig. 2: mIDA-induced $\mathrm{Ca}^{2+}$ and ROS release in Arabidopsis}

a, Normalized R-GECO1 fluorescence intensities $(\Delta \mathrm{F} / \mathrm{F})$ were measured from regions of interest (ROI) (outlined in red) in the meristematic and elongation zone of the root. Shown are cytosolic calcium concentration $\left(\left[\mathrm{Ca}^{2+}\right]_{\mathrm{cyt}}\right.$ ) dynamics in the ROI in response to $1 \mu \mathrm{M}$ mIDA over time. (see also Movie 1). Representative response from 10 roots. Red arrow at 0 minutes (min) indicates application of mIDA peptide. b, Representative response from 10 roots. The increase in $\left[\mathrm{Ca}^{2+}\right]$ cyt response propagates through the roots as two waves seen as normalized R-GECO1 fluorescence intensities $(\triangle \mathrm{F} / \mathrm{F})$ shown as a heat map. c, Expression of pHAE:Venus-H2B and $p H S L 2:$ Venus-H2B in 7 daysold roots. Scale bar $=50 \mu \mathrm{m}$, single plane image, magenta $=$ propidium iodide stain. $\mathbf{d}$, Increase in $\left[\mathrm{Ca}^{2+}\right]_{\text {cyt }}$ in seedlings expressing the cytosolic localized Aequorin-based luminescence $\mathrm{Ca}^{2+}$ sensor (Aeq) measured in relative light units (RLU) treated with $1 \mu \mathrm{M}$ mIDA (black). No response is observed in Aeq seedlings treated with $1 \mu \mathrm{M} \mathrm{IDA}^{\Delta \mathrm{N} 69}$ (gray) or in hae hsl2 Aeq seedlings treated with $1 \mu \mathrm{M}$ mIDA (blue). e, Increase in $\left[\mathrm{Ca}^{2+}\right]_{\mathrm{cyt}}$ in Aeq expressing flowers at position 5 and 6 (See Supplementary Fig. 5a for positions) measured in RLU in response to $1 \mu \mathrm{M}$ mIDA. Curves represent average of 3 independent experiments with 4-6 replications in each experiment. f, ROS production detected by the luminol-based assay was monitored over time as RLU. ROS production from hae hsl2 leaf disks expressing 35S:HAE-YFP in response to $1 \mu \mathrm{M}$ mIDA (black) and in WT control (blue).

\section{Fig. 3: ROS production in floral AZs and abscission phenotypes of the rbohd rbohf mutant}

a, ROS production in the AZ (white arrow head) detected by using the fluorescent dye $\mathrm{H}_{2} \mathrm{DCF}$ DA in WT, hae hsl2 and rbohd rbohf flowers at position 6 (See Supplementary Fig. 5a for positions). Scale bar $=100 \mu \mathrm{m}$, maximum intensity projections of z-stacks. Representative pictures from 9 flowers. b, Representative pictures of WT, hae hsl2 and rbohd rbohf inflorescences, notice that only hae hsl2 retains floral organs along the inflorescence as seen by arrows in inserts, scale bar for inflorescences $=1 \mathrm{~cm}$. c, Petal break strength $(\mathrm{pBS})$ measurements of WT and the rbohd rbohf mutant at position 1-6 along the inflorescence. Force required to remove petals from the receptacle represented in gram equivalent. $*$ = significantly different from $\mathrm{WT}$ at the given position ( $\mathrm{p}<0.05$, student t-test, two tailed). 
Fig. 4: Promoter activity of $H S L 2, R L K 7, H A E$ and $F L S 2$ in cells undergoing cell separation a, Microscopic analysis of 7-days-old pHSL2:Venus-H2B, pRLK7:Venus-H2B, pHAE:Venus-H2B and $p F L S 2:$ Venus $-H 2 B$ roots. Fluorescent nuclei could be observed during emergence of lateral roots (arrows indicate LR). Representative pictures of $\mathrm{n}=8$, scale bar $=50 \mu \mathrm{m}$, single plane image. b, Microscopic analysis of pHSL2:Venus-H2B, pRLK7:Venus-H2B, pHAE:Venus-H2B and pFLS2:Venus-H2B flowers at position 6 (See Supplementary Fig. 5a for positions). Fluorescent nuclei could be observed in the AZ (arrows indicate AZ). Representative pictures of $n=8$, scale bar $=100 \mu \mathrm{m}$, maximum intensity projections of z-stacks.

\section{Fig. 5: HSL2 interaction with RLK7 is promoted by the presence of mIDA and PIP1.}

a, Receptor interaction shown by FRET-APB. Receptors of interest are fused to either an acceptor (mCherry) or donor (GFP) fluorophore and expressed in N.benthamiana. Protein-protein interactions are measured as changes in the GFP emission after photobleaching of mCherry, presented as $\mathrm{E}^{\mathrm{FRET}}[\%]$. Donor-only samples were used as negative controls. A RLK7-GFPmCherry construct was used as a positive control. Line at $4 \%$ indicates GFP background fluctuations. All measurements were performed on $n=15$ cells and experiments were repeated 3 times. b, Representative picture of receptor expression in N.benthamiana leaves, scale bar $=10$ $\mu \mathrm{m}$. c, Plate interaction assays between the extracellular domains (ECDs) of HSL2 (bait) and RLK7 (prey) in the absence (No treatment) and presence of $1 \mu \mathrm{M}$ mIDA, PIP1, IDA ${ }^{\Delta N 69}$, and elf18 represented as cumulative absorbance (Abs $650 \mathrm{~nm}$ ) over $10 \mathrm{~h}$. Dots represent individual observations at each minute from four technical replicates. Box plots display the $1^{\text {st }}$ and $3^{\text {rd }}$ quartiles, split by the median; the whiskers are drawn down to the $10^{\text {th }}$ percentile and up to the 90 th. $* * * *=$ significantly different from non-treated sample $(\mathrm{p}<0.0001)$.

Fig. 6: mIDA induced expression of defense-associated marker genes in $h s l 2$, $r l k 7$ and $h s l 2 r l k 7$ mutants

RT-qPCR data showing transcription of FRK1, MYB51, and PEP3 in WT, hsl2, rlk7 and hsl2 rlk7 seedlings exposed to $1 \mu \mathrm{M}$ mIDA for $1 \mathrm{~h}$ compared to untreated seedlings (control). ACTIN was used to normalize mRNA levels. Figure represent 3 biological replicates with 4 technical replicates. $*=$ Significantly different to untreated of same genotype, ${ }^{*}=$ Significantly different to untreated WT (all untreated samples tested), ${ }^{* * *}=$ Significantly different to mIDA treated WT (all IDA treated samples tested). ( $\mathrm{p}<0.05$, student t-test, two tailed). Statistical analyses are presented in Supplementary Table 3. 


\section{Fig. 7: IDA regulates cell separation and modulates immunity}

IDA and the IDL peptides control cell separation processes during plant development and in response to abiotic and biotic stress ${ }^{7,14,19,24}$. The regulation of IDA expression is under developmental control but can also be activated by abiotic and biotic stimuli. During developmental control of floral organ abscission and emergence of lateral roots IDA relays a signal through receptor complexes including HAE, HSL2, SERK1 and BAK1 to modulate the expression of cell wall remodeling (CWR) genes. In cells undergoing cell separation IDA and PIP1 can promote interaction of HSL2 and RLK7 to modulate expression of defense-associated genes thereby mounting a cell specific immune response. MAMPs and DAMPs activating the FLS2 and RLK7 receptors lead to an induction of defenseassociated genes and IDA, which in turn will enhance HSL2 and RLK7 signaling. Receptor complexes regulating cell separation and immunity share similar signaling components. Both activation of receptors modulating cell separation and defense stimulate ROS release and an increase in cytosolic $\mathrm{Ca}^{2+}$ although the mechanism for ROS and $\mathrm{Ca}^{2+}$ release appears to be different.

\section{Movies}

Movie 1: $\left[\mathrm{Ca}^{2+}\right]_{\text {cyt }}$ dynamics in R-GECO1 expressing root tip in response to $1 \mu \mathrm{M}$ mIDA. 10 daysold roots were treated with $1 \mu \mathrm{M}$ mIDA and changes in $\left[\mathrm{Ca}^{2+}\right]_{\text {cyt }}$ was recorded over time. Response shown as normalized fluorescence intensities $(\Delta \mathrm{F} / \mathrm{F})$. Images were recorded with a frame rate of 5 seconds. Movie corresponds to measurements shown in Fig. 2a,b. Representative response from 10 roots. Time shown as, hh:mm:ss.

Movie 2: $\left[\mathrm{Ca}^{2+}\right]_{\text {cyt }}$ dynamics in R-GECO1 expressing root tip in response to $1 \mu \mathrm{M}$ flg22 10 daysold roots were treated with $1 \mu \mathrm{M}$ flg22 and changes in $\left[\mathrm{Ca}^{2+}\right]_{\text {cyt }}$ was recorded over time. Response shown as normalized fluorescence intensities $(\Delta \mathrm{F} / \mathrm{F})$. Images were recorded with a frame rate of 5 seconds. Movie corresponds to measurements shown in Supplementary Fig. 3. Representative response from 10 roots. Time shown as, hh:mm:ss.

Movie 3: $\left[\mathrm{Ca}^{2+}\right]_{\text {cyt }}$ dynamics in R-GECO1 expressing root tip in response to $1 \mathrm{mM}$ ATP. $1 \mathrm{mM}$ ATP will induce a strong increase in $\left[\mathrm{Ca}^{2+}\right]_{\text {cyt }}$ and was added as a last treatment to all roots acting as a positive control. Response shown as normalized fluorescence intensities $(\Delta \mathrm{F} / \mathrm{F})$. Images were recorded with a frame rate of 5 seconds. Representative response from 10 roots. Time shown as, hh:mm:ss. 

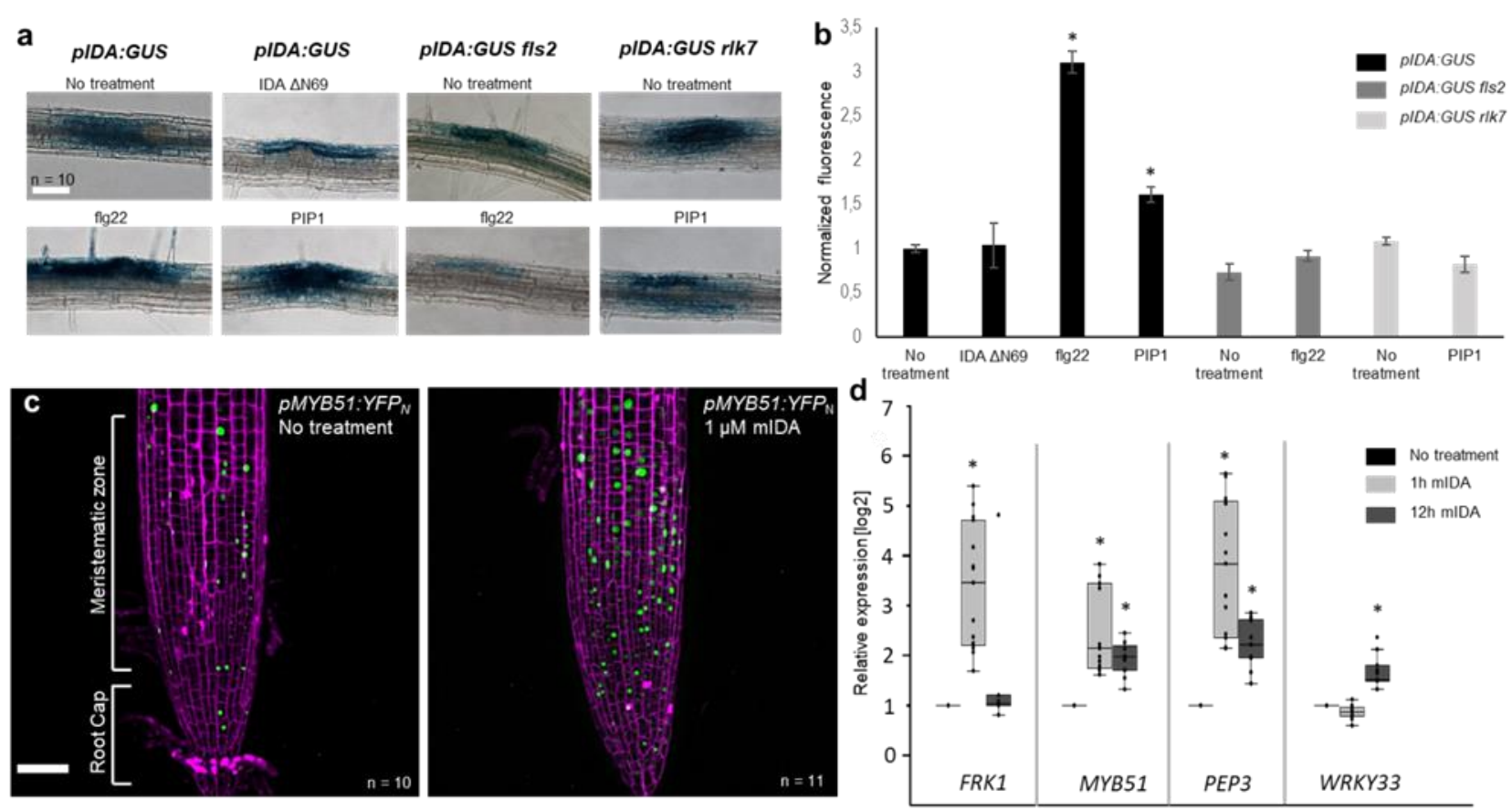

Fig. 1: IDA is induced by biotic stress and triggers defense-associated marker genes

a, Enhanced pIDA:GUS expression after 12 hours (h) treatment with $1 \mu \mathrm{M}$ flg2 22 or $1 \mu \mathrm{M}$ PIP1 is observed in cells surrounding emerging lateral roots. Controls were either not subjected to any stimuli (No treatment) or exposed to $1 \mu \mathrm{M}$ of the inactive IDA peptide, IDA ${ }^{\Delta \mathrm{N} 69} 4,12$. No enhanced flg22 and PIP1 induced expression is observed in pIDA:GUS fls2 or pIDA:GUS rlk7 plants compared to plants grown without the presence of any stimuli (No treatment). Representative picture of $\mathrm{n}=10$, experiment repeated 3 times, scale bar $=50 \mu \mathrm{m}$. b. Normalized emitted fluorescence of fluorochrome 4-methyl umbelliferone (4-MU) in 7 days-old seedlings after $12 \mathrm{~h}$ treatment with $1 \mu \mathrm{M}$ PIP1 and 1 $\mu \mathrm{M}$ flg22 in pIDA:GUS seedlings, pIDA:GUS fls2 seedlings and pIDA:GUS rlk7 seedlings. Controls were either not subjected to any stimuli (No treatment) or exposed to $1 \mu \mathrm{M} \mathrm{IDA}^{\Delta \mathrm{N} 69} \cdot \mathrm{n}=6$, experiment repeated 3 times. ${ }^{*}=$ significantly different from the non-treated (No treatment) sample $(\mathrm{p}<0.05$, student t-test, two tailed). c, $p M Y B 51: Y F P_{N}$ expression is enhanced in roots after $7 \mathrm{~h}$ exposure to 1 $\mu \mathrm{M}$ mIDA peptide compared to untreated roots (control), scale bar $=50 \mu \mathrm{m}$, maximum intensity projections of z-stacks, magenta = propidium iodide stain. d, Transcripts of FRK1, MYB51, PEP3 and WRKY33 in WT seedlings exposed to $1 \mu \mathrm{M}$ mIDA for $1 \mathrm{~h}$ (light gray) and to $1 \mu \mathrm{M}$ mIDA for 12 h (dark gray) compared to untreated tissue (black). RNA levels were measured by RT-qPCR analysis. ACTIN was used to normalize mRNA levels. $\mathrm{n}=4$, three biological replicates. $*=$ significantly different from untreated sample ( $p<0.05$, student t-test, two tailed). Statistical analyses are presented in Supplementary Table 3. 

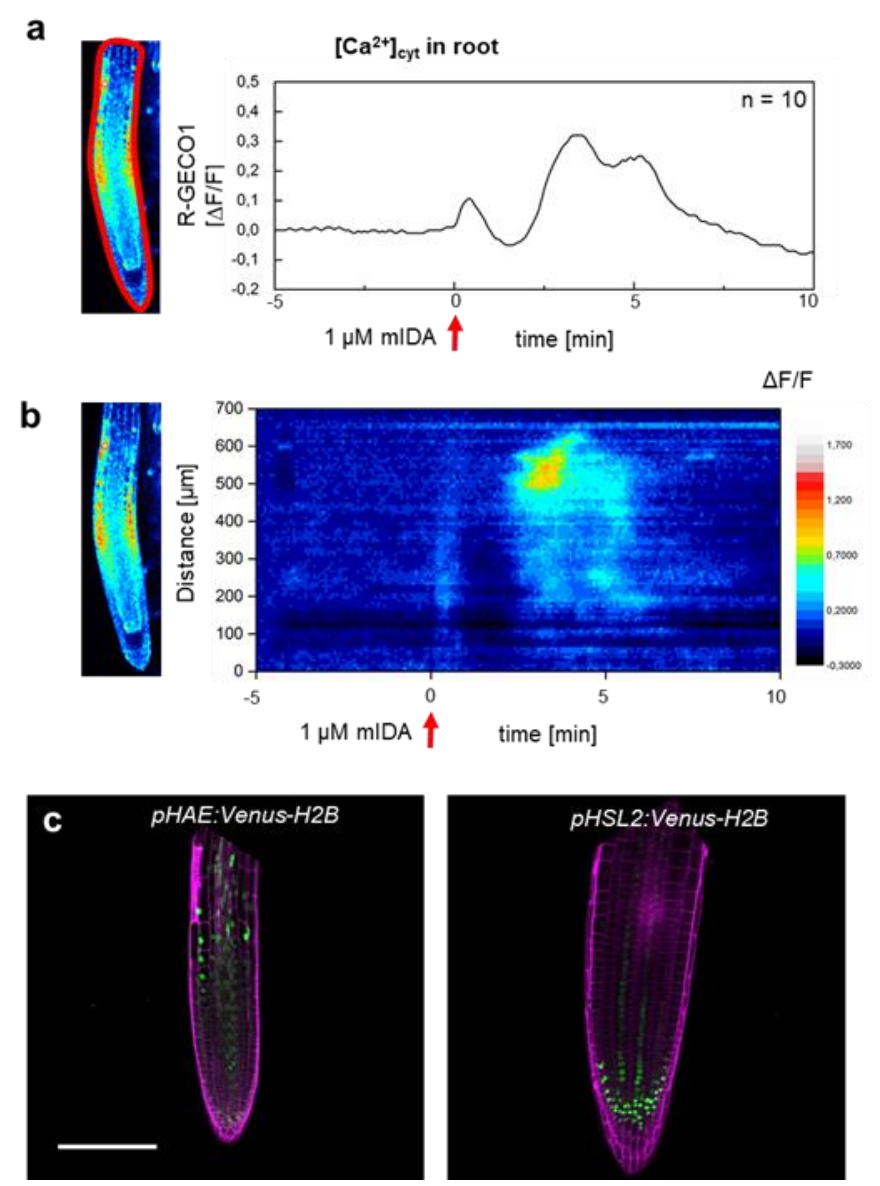
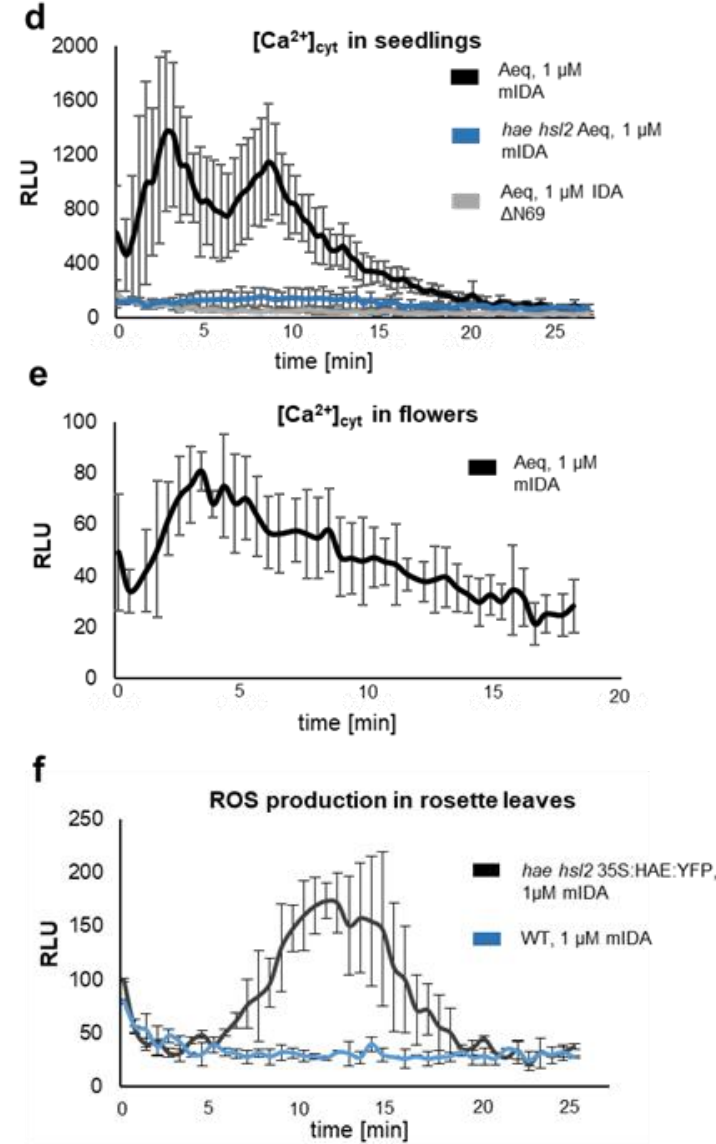

\section{Fig. 2: mIDA-induced $\mathrm{Ca}^{2+}$ and $\mathrm{ROS}$ release in Arabidopsis}

a, Normalized R-GECO1 fluorescence intensities $(\Delta \mathrm{F} / \mathrm{F})$ were measured from regions of interest (ROI) (outlined in red) in the meristematic and elongation zone of the root. Shown are cytosolic calcium concentration $\left(\left[\mathrm{Ca}^{2+}\right]_{\mathrm{cyt}}\right.$ ) dynamics in the ROI in response to $1 \mu \mathrm{M}$ mIDA over time. (see also Movie 1). Representative response from 10 roots. Red arrow at 0 minutes (min) indicates application of mIDA peptide. $\mathbf{b}$, Representative response from 10 roots. The increase in $\left[\mathrm{Ca}^{2+}\right]$ cyt response propagates through the roots as two waves seen as normalized R-GECO1 fluorescence intensities $(\triangle \mathrm{F} / \mathrm{F})$ shown as a heat map. c, Expression of pHAE:Venus-H2B and $p H S L 2:$ Venus-H2B in 7 daysold roots. Scale bar $=50 \mu \mathrm{m}$, single plane image, magenta $=$ propidium iodide stain. $\mathbf{d}$, Increase in $\left[\mathrm{Ca}^{2+}\right]_{\mathrm{cyt}}$ in seedlings expressing the cytosolic localized Aequorin-based luminescence $\mathrm{Ca}^{2+}$ sensor (Aeq) measured in relative light units (RLU) treated with $1 \mu \mathrm{M}$ mIDA (black). No response is observed in Aeq seedlings treated with $1 \mu \mathrm{M} \mathrm{IDA}^{\Delta \mathrm{N} 69}$ (gray) or in hae hsl2 Aeq seedlings treated with $1 \mu \mathrm{M}$ mIDA (blue). e, Increase in $\left[\mathrm{Ca}^{2+}\right]_{\mathrm{cyt}}$ in Aeq expressing flowers at position 5 and 6 (See Supplementary Fig. 5a for positions) measured in RLU in response to $1 \mu \mathrm{M}$ mIDA. Curves represent average of 3 independent experiments with 4-6 replications in each experiment. f, ROS production 
bioRxiv preprint doi: https://doi.org/10.1101/761346; this version posted September 8, 2019. The copyright holder for this preprint (which was not certified by peer review) is the author/funder, who has granted bioRxiv a license to display the preprint in perpetuity. It is made available under aCC-BY-NC-ND 4.0 International license.

detected by the luminol-based assay was monitored over time as RLU. ROS production from hae hsl2 leaf disks expressing 35S:HAE-YFP in response to $1 \mu \mathrm{M}$ mIDA (black) and in WT control (blue). 

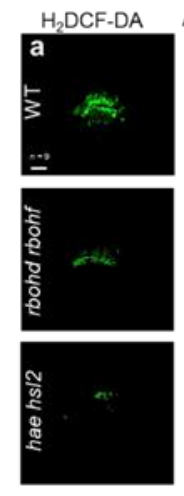
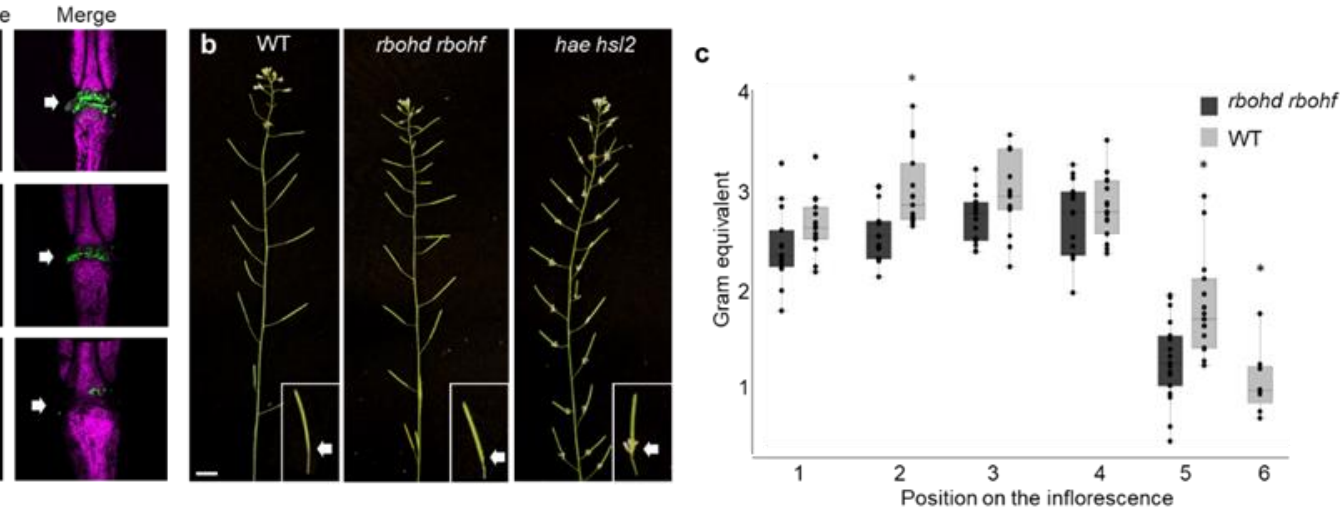

Fig. 3: ROS production in floral AZs and abscission phenotypes of the rbohd rbohf mutant

a, ROS production in the AZ (white arrow head) detected by using the fluorescent dye $\mathrm{H}_{2} \mathrm{DCF}$ DA in WT, hae hsl2 and rbohd rbohf flowers at position 6 (See Supplementary Fig. 5a for positions). Scale bar $=100 \mu \mathrm{m}$, maximum intensity projections of z-stacks. Representative pictures from 9 flowers. b, Representative pictures of WT, hae hsl2 and rbohd rbohf inflorescences, notice that only hae hsl2 retains floral organs along the inflorescence as seen by arrows in inserts, scale bar for inflorescences $=1 \mathrm{~cm}$. c, Petal break strength $(\mathrm{pBS})$ measurements of WT and the rbohd rbohf mutant at position 1-6 along the inflorescence. Force required to remove petals from the receptacle represented in gram equivalent. $*=$ significantly different from $\mathrm{WT}$ at the given position ( $\mathrm{p}<0.05$, student t-test, two tailed). 
a Emerging lateral roots
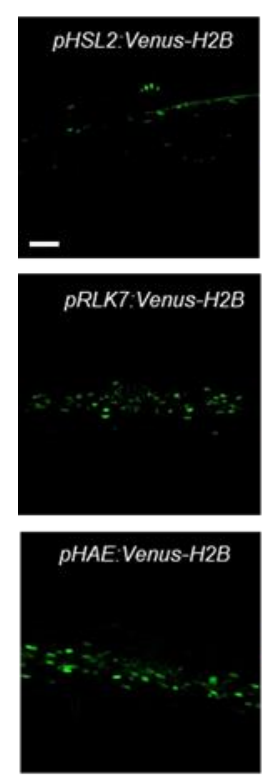

pFLS2:Venus-H2B
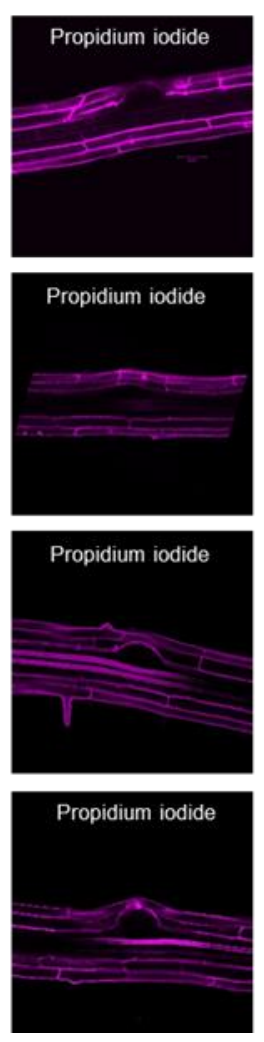
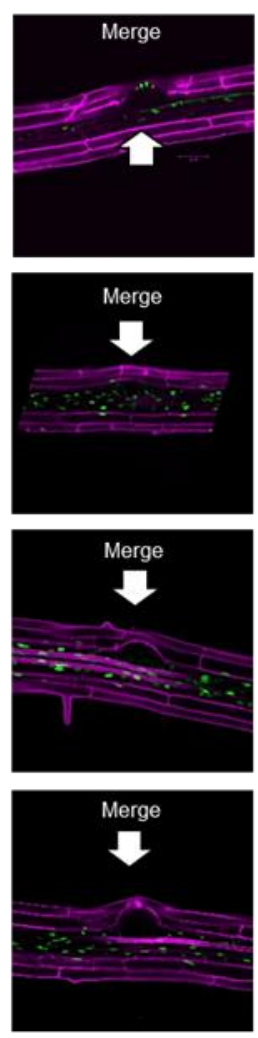

b Abscission zone, floral position 7
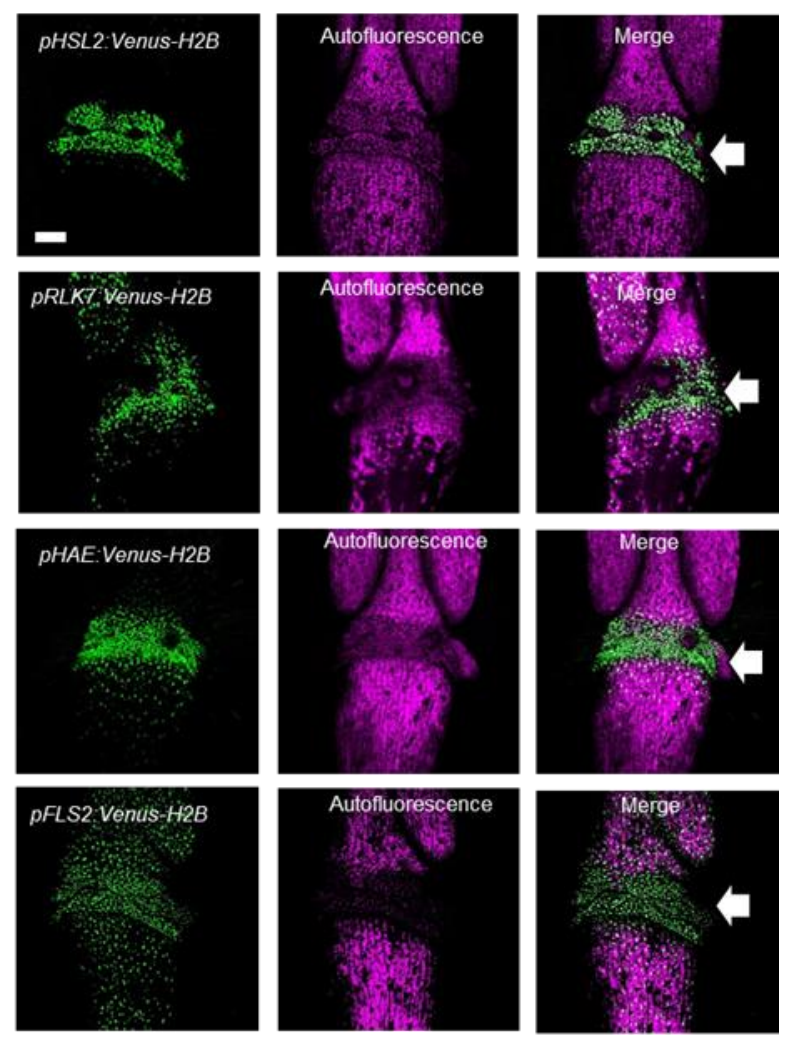

Fig. 4: Promoter activity of $H S L 2, R L K 7, H A E$ and $F L S 2$ in cells undergoing cell separation a, Microscopic analysis of 7-days-old pHSL2:Venus-H2B, pRLK7:Venus-H2B, pHAE:Venus-H2B and $p F L S 2:$ Venus $-H 2 B$ roots. Fluorescent nuclei could be observed during emergence of lateral roots (arrows indicate LR). Representative pictures of $\mathrm{n}=8$, scale bar $=50 \mu \mathrm{m}$, single plane image. b, Microscopic analysis of pHSL2:Venus-H2B, pRLK7:Venus-H2B, pHAE:Venus-H2B and pFLS2:Venus-H2B flowers at position 6 (See Supplementary Fig. 5a for positions). Fluorescent nuclei could be observed in the AZ (arrows indicate AZ). Representative pictures of $n=8$, scale bar $=100 \mu \mathrm{m}$, maximum intensity projections of z-stacks. 

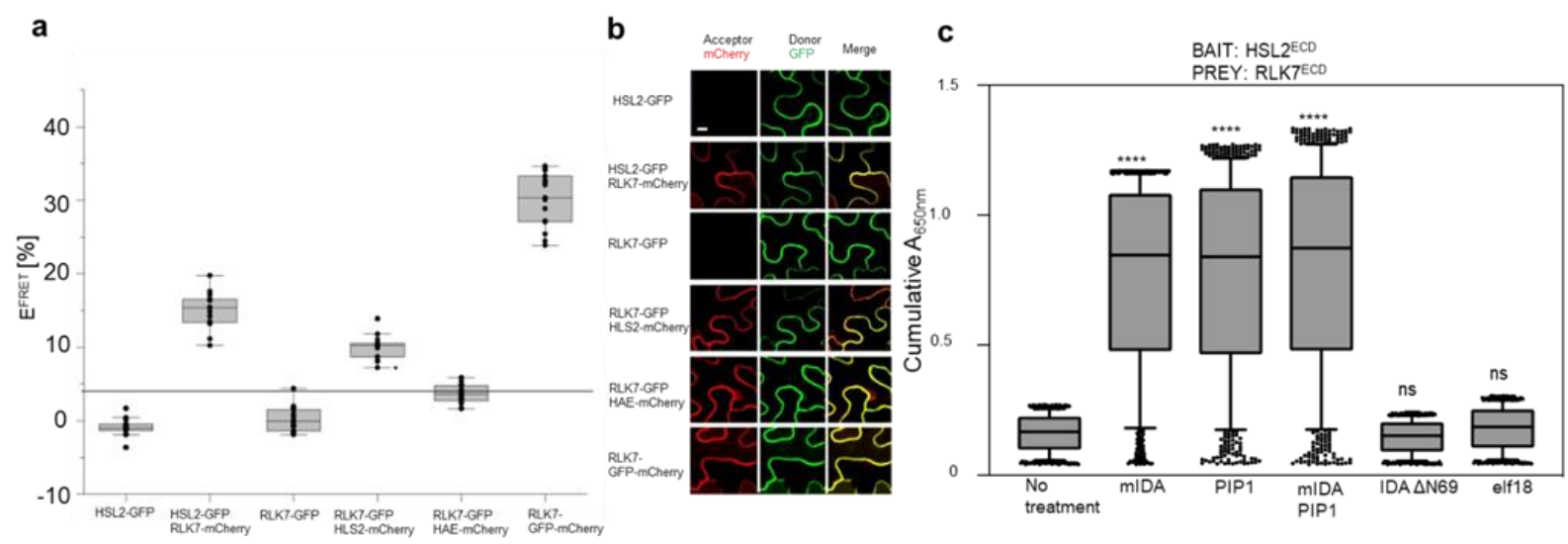

Fig. 5: HSL2 interaction with RLK7 is promoted by the presence of mIDA and PIP1.

a, Receptor interaction shown by FRET-APB. Receptors of interest are fused to either an acceptor (mCherry) or donor (GFP) fluorophore and expressed in N.benthamiana. Protein-protein interactions are measured as changes in the GFP emission after photobleaching of mCherry, presented as $\mathrm{E}^{\mathrm{FRET}}[\%]$. Donor-only samples were used as negative controls. A RLK7-GFPmCherry construct was used as a positive control. Line at $4 \%$ indicates GFP background fluctuations. All measurements were performed on $n=15$ cells and experiments were repeated 3 times. b, Representative picture of receptor expression in N.benthamiana leaves, scale bar $=10$ $\mu \mathrm{m}$. c, Plate interaction assays between the extracellular domains (ECDs) of HSL2 (bait) and RLK7 (prey) in the absence (No treatment) and presence of $1 \mu \mathrm{M}$ mIDA, PIP1, IDA ${ }^{\Delta \mathrm{N} 69}$, and elf18 represented as cumulative absorbance (Abs $650 \mathrm{~nm}$ ) over $10 \mathrm{~h}$. Dots represent individual observations at each minute from four technical replicates. Box plots display the $1^{\text {st }}$ and $3^{\text {rd }}$ quartiles, split by the median; the whiskers are drawn down to the $10^{\text {th }}$ percentile and up to the 90 th. $* * * *=$ significantly different from non-treated sample $(\mathrm{p}<0.0001)$. 


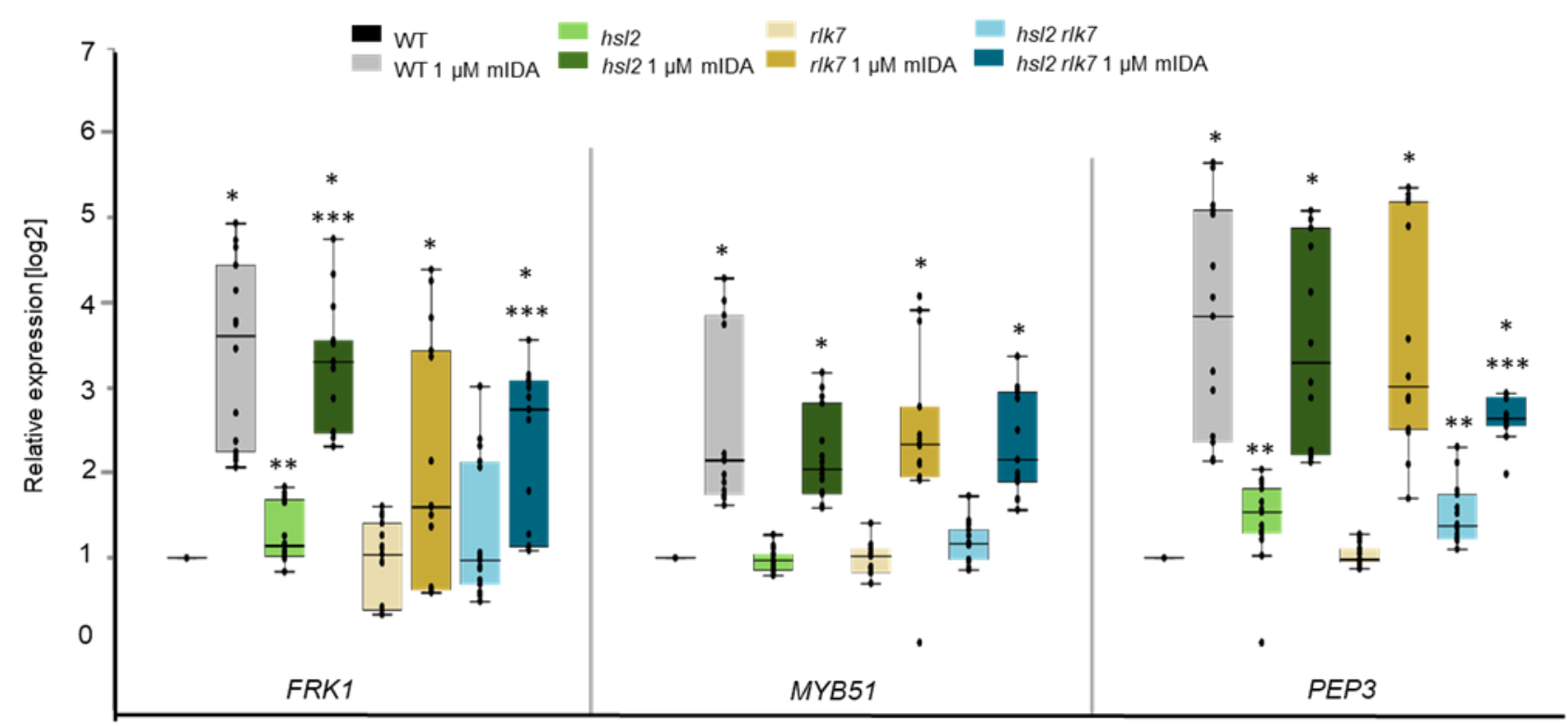

Fig. 6: mIDA induced expression of defense-associated marker genes in $h s l 2, r l k 7$ and $h s l 2 r l k 7$ mutants

RT-qPCR data showing transcription of FRK1, MYB51, and PEP3 in WT, hsl2, rlk7 and hsl2 rlk7 seedlings exposed to $1 \mu \mathrm{M}$ mIDA for $1 \mathrm{~h}$ compared to untreated seedlings (control). ACTIN was used to normalize mRNA levels. Figure represent 3 biological replicates with 4 technical replicates. $*=$ Significantly different to untreated of same genotype, $* *=$ Significantly different to untreated WT (all untreated samples tested), *** = Significantly different to mIDA treated WT (all IDA treated samples tested). ( $\mathrm{p}<0.05$, student t-test, two tailed). Statistical analyses are presented in Supplementary Table 3. 


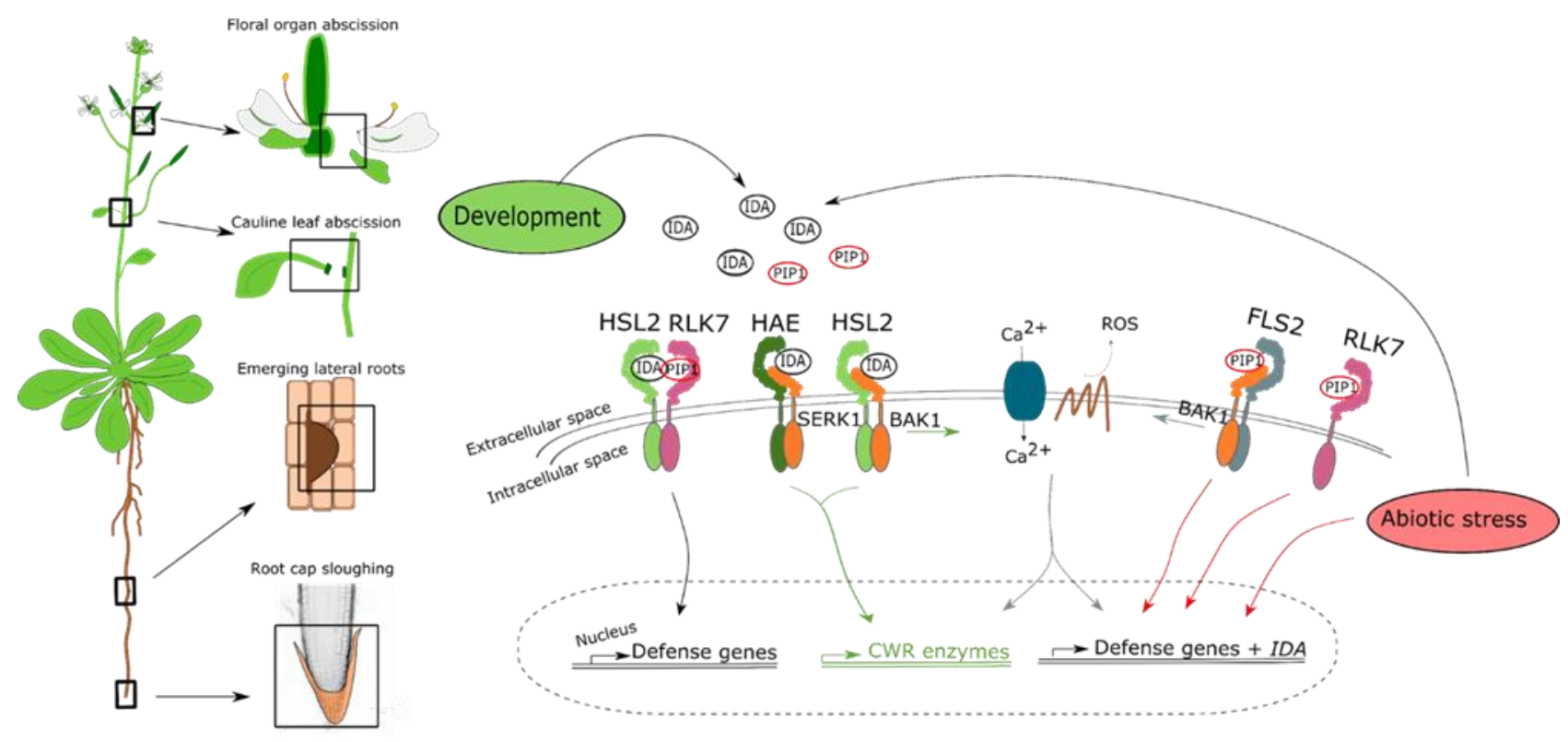

\section{Fig. 7: IDA regulates cell separation and modulates immunity}

IDA and the IDL peptides control cell separation processes during plant development and in response to abiotic and biotic stress ${ }^{7,14,19,24}$. The regulation of IDA expression is under developmental control but can also be activated by abiotic and biotic stimuli. During developmental control of floral organ abscission and emergence of lateral roots IDA relays a signal through receptor complexes including HAE, HSL2, SERK1 and BAK1 to modulate the expression of cell wall remodeling (CWR) genes. In cells undergoing cell separation IDA and PIP1 can promote interaction of HSL2 and RLK7 to modulate expression of defense-associated genes thereby mounting a cell specific immune response. MAMPs and DAMPs activating the FLS2 and RLK7 receptors lead to an induction of defenseassociated genes and IDA, which in turn will enhance HSL2 and RLK7 signaling. Receptor complexes regulating cell separation and immunity share similar signaling components. Both activation of receptors modulating cell separation and defense stimulate ROS release and an increase in cytosolic $\mathrm{Ca}^{2+}$ although the mechanism for ROS and $\mathrm{Ca}^{2+}$ release appears to be different. 